\title{
A Multibit Trie Structure Designed for Peer-to-Peer (P2P) Live Video Streaming
}

\author{
by \\ Boyuan Zhang B.Eng \\ Carleton University \\ A thesis submitted to the Faculty of Graduate Studies and Research \\ in partial fulfillment of the requirements for the degree of \\ Master of Applied Science in Electrical Engineering \\ Ottawa-Carleton Institute for Electrical and Computer Engineering (OCIECE) \\ Department of Systems and Computer Engineering \\ Carleton University \\ Ottawa, Ontario, Canada, K1S 5B6
}

November 2011

(C) Copyright 2011, Boyuan Zhang 
Library and Archives

Canada

Published Heritage

Branch

395 Wellington Street

Ottawa ON K1A ON4

Canada
Bibliothèque et

Archives Canada

Direction du

Patrimoine de l'édition

395 , rue Wellington

Ottawa ON K1A ON4

Canada
Your file Votre référence

ISBN: 978-0-494-87828-6

Our file Notre référence

ISBN: $978-0-494-87828-6$

\section{NOTICE:}

The author has granted a nonexclusive license allowing Library and Archives Canada to reproduce, publish, archive, preserve, conserve, communicate to the public by telecommunication or on the Internet, loan, distrbute and sell theses worldwide, for commercial or noncommercial purposes, in microform, paper, electronic and/or any other formats.

The author retains copyright ownership and moral rights in this thesis. Neither the thesis nor substantial extracts from it may be printed or otherwise reproduced without the author's permission.
AVIS:

L'auteur a accordé une licence non exclusive permettant à la Bibliothèque et Archives Canada de reproduire, publier, archiver, sauvegarder, conserver, transmettre au public par télécommunication ou par l'Internet, prêter, distribuer et vendre des thèses partout dans le monde, à des fins commerciales ou autres, sur support microforme, papier, électronique et/ou autres formats.

L'auteur conserve la propriété du droit d'auteur et des droits moraux qui protege cette thèse. $\mathrm{Ni}$ la thèse ni des extraits substantiels de celle-ci ne doivent être imprimés ou autrement reproduits sans son autorisation.
In compliance with the Canadian Privacy Act some supporting forms may have been removed from this thesis.

While these forms may be included in the document page count, their removal does not represent any loss of content from the thesis.
Conformément à la loi canadienne sur la protection de la vie privée, quelques formulaires secondaires ont été enlevés de cette thèse.

Bien que ces formulaires aient inclus dans la pagination, il n'y aura aucun contenu manquant. 
The undersigned recommend to the Faculty of Graduate Studies and Research acceptance of the thesis

\title{
A Multibit Trie Structure Designed for Peer-to-Peer (P2P) Live Video Streaming
}

\author{
submitted by \\ Boyuan Zhang, B.Eng \\ in partial fulfillment of the requirements for the degree of \\ Master of Applied Science in Electrical Engineering
}

Howard Schwartz, Chair, Department of Systems and Computer Engineering

Changcheng Huang, Thesis Supervisor

James Yan, Thesis Co-Supervisor

Carleton University

November 2011 


\begin{abstract}
This thesis addresses the problem of how to develop an efficient Peer-to-Peer (P2P) architecture that improves the performance of P2P live video streaming (LVS) application. Current P2P LVS software causes problems such as long start-up delay and non-smooth playback for end users. Based on a carefully analysis of the advantages/disadvantages of previous research and the behavior of users during a P2P live streaming session, a trie structure called Bottom-Up Trie (BU-Trie) is proposed for content distribution. We demonstrate that the main benefits of BU-Trie are to minimize the start-up delay, localize traffic, and improve both bandwidth and content bottleneck. We also propose a new chunk selection algorithm called Most Popular Chunk First (MPCF). Our simulation results show that, by applying the MPCF algorithm, the sequential throughput of downloaded chunks is increased compared to other approaches. Furthermore we verify our simulation results with mathematical analysis.
\end{abstract}


To my parents, Guoli Zhang and Ying Wang, and my fiancée: Si Lao 咸给我政爱的父母和末婚事 


\section{Acknowledgements}

I would like to thank my thesis supervisor, Professor C. Huang, and co-supervisor, Professor James Yan for their guidance and encouragement throughout the period of my study. This work was supported by NSERC Grant CRDPJ 354729-07. 


\section{Table of Contents}

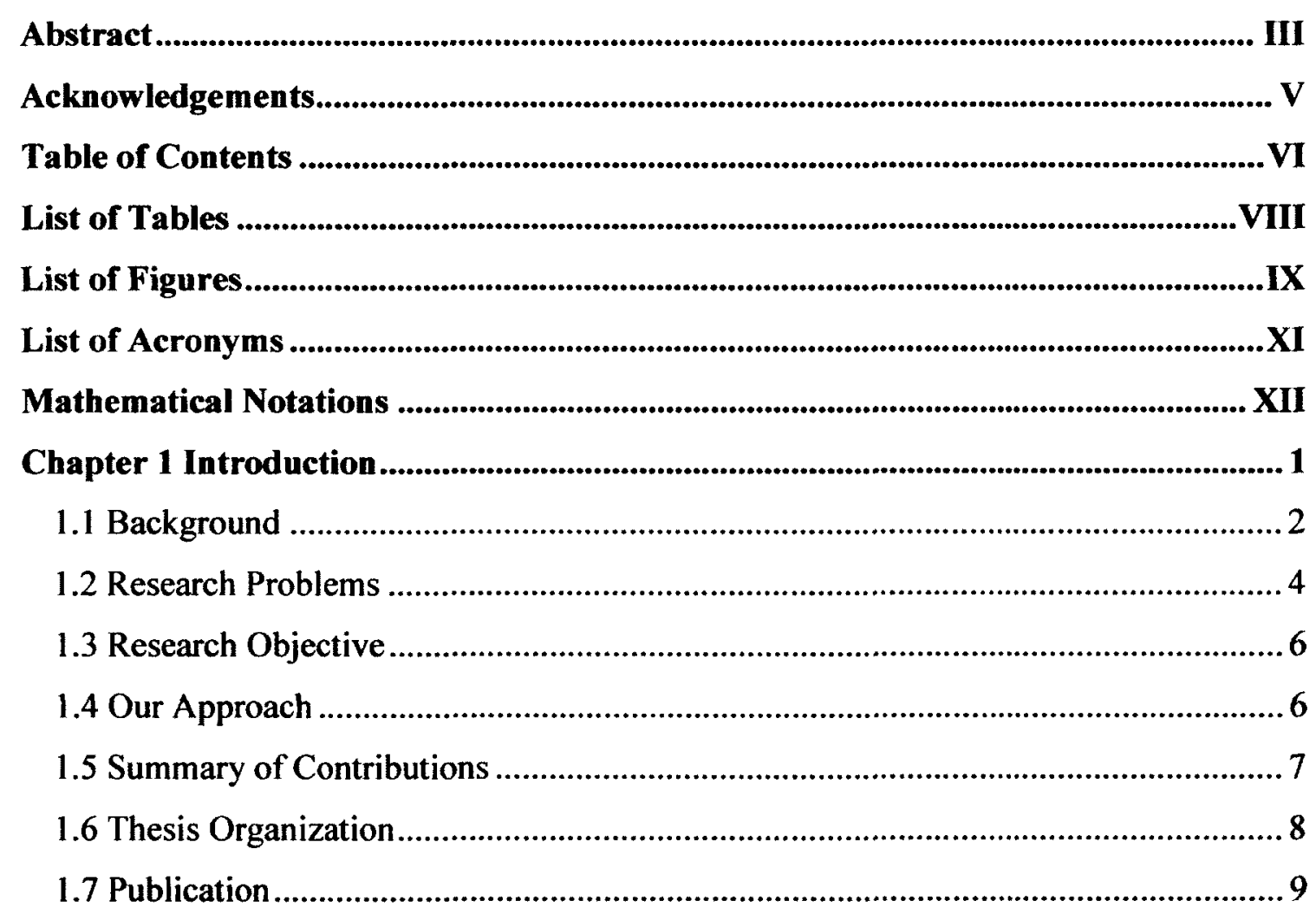

Chapter 2 Background Knowledge ................................................................................... 10

2.1 Review of Peer-to-Peer Live Video Streaming (P2P LVS) .................................... 10

2.1.1 Path-driven algorithms................................................................................. 12

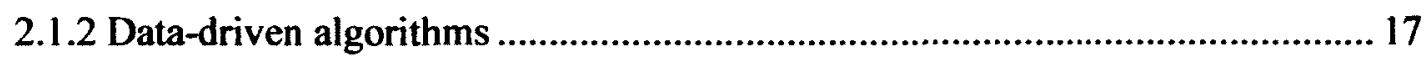

2.1.3 Hybrid Algorithms ....................................................................................... 19

2.2 Chunk Selection Strategy ................................................................................ 20

2.2.1 Chunk Selection Algorithms..................................................................... 20

2.2.2 Related Researches ..................................................................................... 23

Chapter 3 Overall Structure of BU-Trie................................................................................. 24 


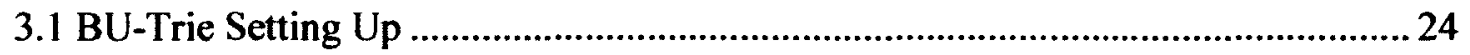

3.2 Two Phases Design Approach.......................................................................... 29

Chapter 4 Two Phases of BU-Trie......................................................................................31

4.1 Diffusion Phase of BU-Trie .............................................................................. 31

4.2 Objective of Swarming Phase ............................................................................... 34

4.3 Swarming Phase Design and MPCF Algorithm ...................................................... 36

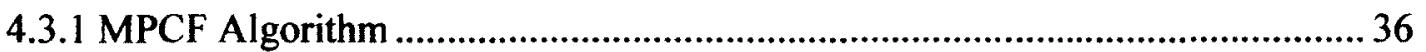

4.3.2 Mathematical Model of MPCF .................................................................... 40

4.3.3 Swarming Phase Design with MPCF ............................................................. 49

Chapter 5 Simulations and Performance Evaluations .................................................... 54

5.1 Network Model......................................................................................................... 54

5.2 Sequential Throughput Test ............................................................................. 57

5.2.1 Bottom-Up Trie (BU-Trie) .......................................................................... 57

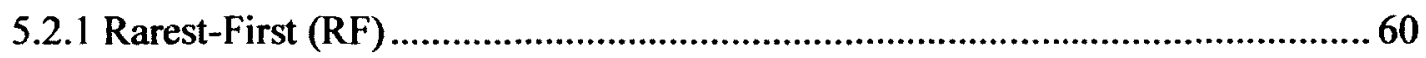

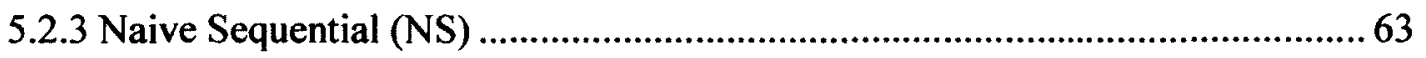

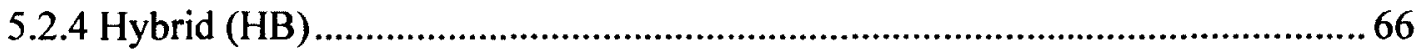

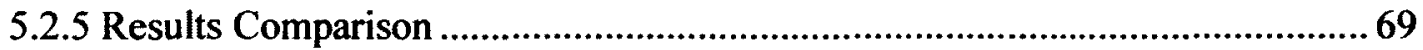

5.3 End-to-End Delay Test ......................................................................................... 76

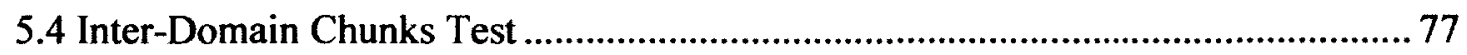

Chapter 6 Conclusion ............................................................................................. 79

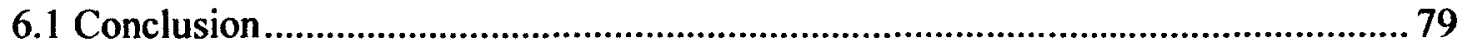

6.2 Suggestions for Future Work ........................................................................... 81

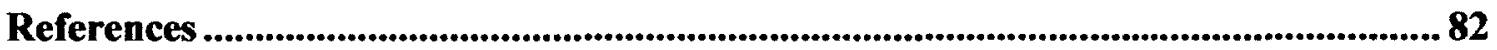




\section{List of Tables}

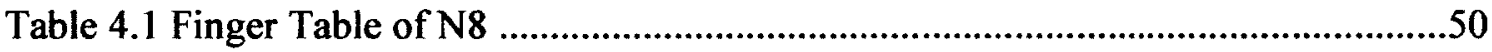

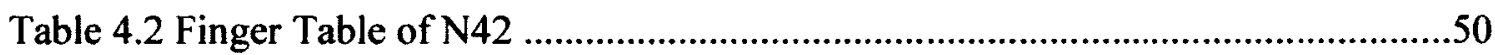

Table 5.1 Simulation Results and Confidential Interval (BU-Trie) ..................60

Table 5.2 Simulation Results and Confidential Interval (RF) ................................63

Table 5.3 Simulation Results and Confidential Interval $(s=1)$........................66

Table 5.4 Simulation Results and Confidential Interval (HB) .......................69

Table 5.5 Sequential Throughput Test Results (Comparison) ........................71

Table 5.6 The Average Probability of Downloading $1^{\text {st }}$ Missing Chunk in Each Round .72

Table 5.7 Simulation Results and Confidential Interval (BU-Trie) ...................74

Table 5.8 Average Number of Inter-Domain Chunks .............................78 


\section{List of Figures}

Figure 0.1: A High Level View of P2P Network Architecture ........................

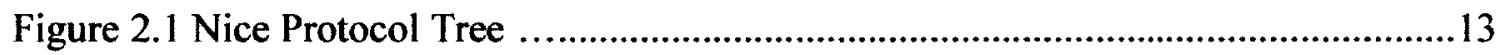

Figure 2.2 Organization of Peers in ZIGZAG................................... 14

Figure 2.3 Data Delivery Topology of ZIGZAG..............................

Figure 2.4 MDC Sub-streams of Splitstream ................................... 17

Figure 3.1 Bottom-Up Trie Architecture .........................................25

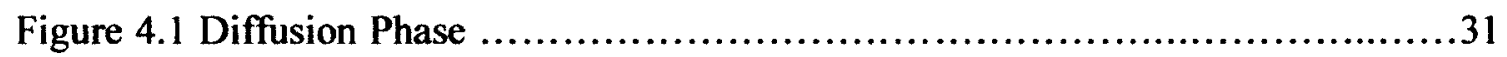

Figure 4.2 Example of Peer's Bitmap after Diffusion Phase ......................... 32

Figure 4.3 Example of Downloading Missing Chunks in Swarming Phase ....................33

Figure 4.4 Rarest-First Chunks Selection Algorithm...........................35

Figure 4.5 Naive Sequential Chunks Selection Algorithm........................36

Figure 4.6 Most Popular Chunk First (MPCF) Chunks Selection Algorithm........37

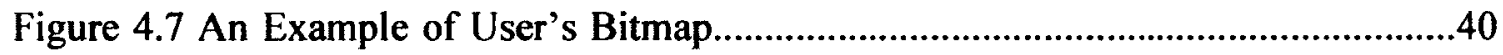

Figure 4.8 An Example of New Joined User's Bitmap..............................................43

Figure 4.9 User's Bitmap after Downloading...................................44

Figure 4.10 User's Bitmap after Downloading the 2nd Chunk in Round 1...................44

Figure 4.11 Sequentiality Vs. Number of Downloaded Chunks............................47

Figure 4.12 Bitmap (Key=54) Locating Process by Node 8 (N8) in Local Network........49 
Figure 4.13 An Example of The Shared Bitmap........................................................51

Figure 4.14 An Example of The Individual Bitmap of N8 ........................................51

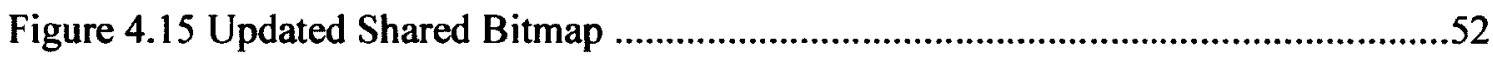

Figure 4.16 Updated Individual Bitmap of N8.....................................................52

Figure 5.1 Network Topology for Simulation ......................................55

Figure 5.2 BU-Trie Structure for Simulation ..................................... 56

Figure 5.3 Number of Packets Received Vs. Time (BU-Trie) $\ldots \ldots \ldots \ldots \ldots \ldots \ldots \ldots \ldots . \ldots . \ldots \ldots$

Figure 5.4 Throughputs and Sequential Throughputs of 32 Peers ...................59

Figure 5.5 Number of Packets Received Vs. Time (BU-Trie) ........................61

Figure 5.6 Throughputs and Sequential Throughputs of 32 Peers (RF) $\ldots \ldots \ldots \ldots \ldots \ldots . \ldots 2$

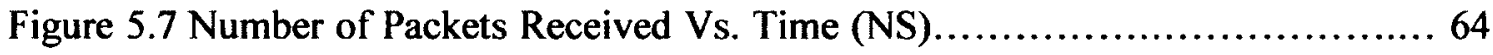

Figure 5.8 Throughputs and Sequential Throughputs of 32 Peers...................65

Figure 5.9 Number of Packets Received Vs. Time (HB).........................67

Figure 5.10 Throughputs and Sequential Throughputs of 32 Peers (HB)..............68

Figure 5.11 Number of chunks Received Vs. Time (Comparison) $\ldots \ldots \ldots \ldots \ldots \ldots \ldots . \ldots$

Figure 5.12 Sequentiality Comparison Test: Math Model Vs. Simulation ..............73

Figure 5.13 Throughputs and Sequential Throughputs of 32 Peers ...................74

Figure 5.14 Sequentiality Comparison Test: Math Model Vs. Simulation ...............75

Figure 5.15 End-to-End Delay of Each Chunk..................................76

Figure 5.16 Number of Inter-Domain Packets...................................78 


\section{List of Acronyms}

\begin{tabular}{ll} 
BU-Trie & Bottom-Up Trie \\
DHT & Distribution Hash Table \\
ETE & End-to-End \\
HB & Hybrid \\
IETF & Internet Engineering Task Force \\
ISP & Internet Service Provider \\
LC & Layered coding \\
LVS & Live Video Streaming \\
MDC & Multiple Description Coding \\
MPCF & Most Popular Chunk First \\
NS & Naive Sequential \\
P2P & Peer-to-Peer \\
RF & Rarest-First \\
STR & Sequential Throughput \\
VOD & Video on Demand \\
\hline
\end{tabular}




\section{Mathematical Notations}

$U_{s}$ - the uploading bandwidth of seed node (bytes/sec)

$U_{p}(i)-$ the uploading bandwidth of normal peer node $i(\mathrm{bytes} / \mathrm{sec})$

$T$ - the average downloading time (sec)

$Y$ - the layer number

$S$ - the sequentiality

$M$ - the number of chunks downloaded

$b$ - the chunk size (bytes)

$n$ - the index of sum equation or product equation

$W(x)$ - the probability that all downloaded chunks are sequential given $x$ chunks are already downloaded.

$P(x)$ - the probability that the $1^{\text {st }}$ missing chunk is downloaded each round.

$f_{j}$ - the normal peer nodes' fraction of uploading bandwidth allocates for chunk $\mathrm{j}$

$N$ - the total number of normal peer nodes in the system.

$T P$ - the average throughput (speed) of downloading video stream (bytes/sec)

$i$ - the index of normal peer nodes

$j$ - the index of chunks 


\section{Chapter 1}

\section{Introduction}

This chapter briefly introduces Peer-to-Peer (P2P) networking, and discusses the different types of applications in P2P networks, especially P2P video streaming. Then, it shows that the network structure and routing algorithm are critical to the performance of $\mathrm{P} 2 \mathrm{P}$ video streaming and the network utilization. The overall performance of $P 2 P$ video streaming is determined by major attributes such as throughput and sequentiality, where throughput is the downloading speed, and sequentiality is the degree to which the file is downloaded sequentially. However, it is proved that these two factors are in a trade-off relationship, since it is impossible to maximize both attributes at the same time. This motivates us to develop an efficient network structure and chunk selection algorithm to improve the overall performance as well as improve the network utilization. We propose a so-called Bottom-Up trie (BU-Tire) structure, which uses traffic localization and a hybrid downloading algorithm to optimize sequential throughput, as well as to minimize the start-up delay in order to improve the overall performance for end users. Finally, the thesis contributions and thesis organization are provided at the end of this chapter. 


\subsection{Background}

A P2P system is a system with completely decentralized self-organization and resource usage. Different from the traditional client and server model, most P2P networks contain only peer nodes which act as both client and server when communicating with other peer nodes in the network. Within a P2P network, peers interact directly with each other in order to share resource and exchange data.

Figure 1.1 gives a high-level view of an unstructured P2P network architecture. In an unstructured P2P architecture, there is no central server. Every node acts as a server and a client at the same time in the network. Both the data and the users' information are distributed among several or all peers. Structured P2P architecture usually has a registration server that response for the registration process, but the contents are still fully distributed among peers. The advantage of P2P networks is that most of the resources are distributed widely or sometimes globally in the network, so a peer can obtain parallel data from other peers who are currently connected to the network. Such a configuration is much more efficient compared to the client/server network. At the same time, the more peers who have certain pieces of data in the network, the better performance of the P2P network will have. Moreover, the effect of single point of failure is reduced since the data is distributed. As a result of these advantages, P2P file sharing systems, such as BitTorrent [1], have become one of the most popular applications in recent years. 


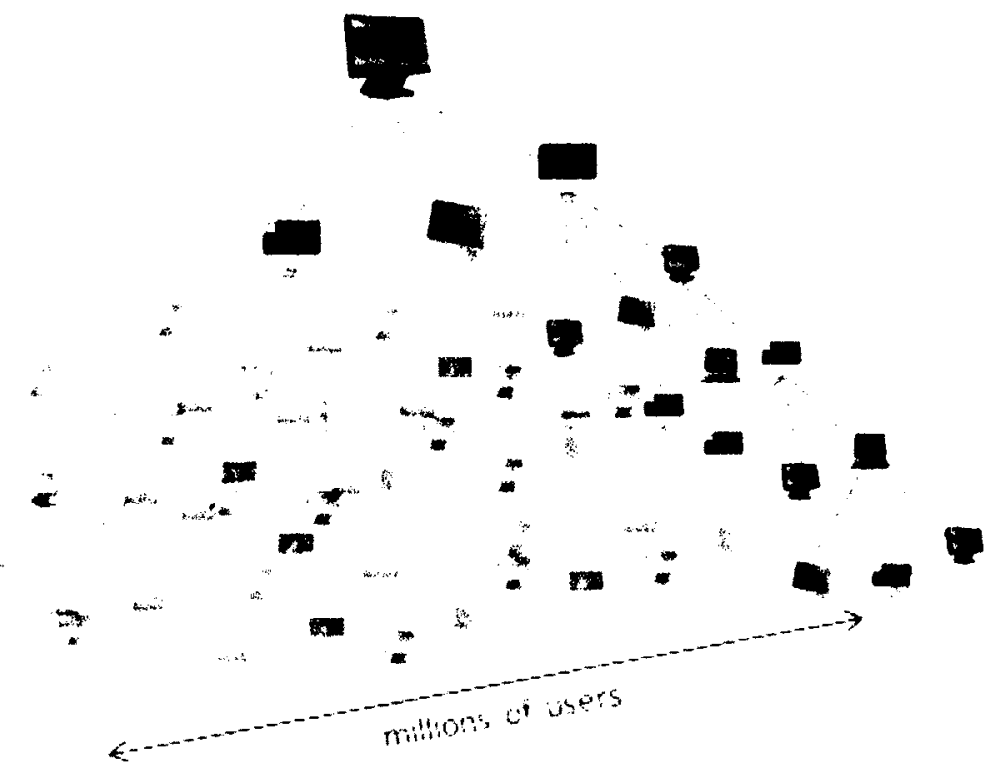

Figure 0.1: A high level view of P2P network architecture

Following the success of P2P file sharing systems, P2P video streaming becomes a more and more popular application in modern Internet and computer communications. There are two types of $\mathrm{P} 2 \mathrm{P}$ video streaming applications that have been widely used: $\mathrm{P} 2 \mathrm{P}$ Video-on-Demand (VOD) and P2P Live Video Streaming (LVS). P2P VOD is where the entire video content has already existed in the network and shared by users during the time users watch this video. On the other hand, P2P LVS is where the video content is being generated by the source at the same time as users watch the video. In other words, the future content of P2P LVS doesn't exist.

P2P VOD applications have been developed for many years. However, due to the high demand for live content, many P2P VOD application providers, such as PPlive [2], UUsee [3], and SopCast [4], have added LVS features to their existing VOD applications in recent years. Many network architectures and algorithms have been developed to 
improve the P2P LVS quality in recent years. In general, those architectures and algorithms can be divided into three categories: path-driven, data-driven algorithms, and hybrid algorithms. Detailed discussions of different types of existing P2P LVS algorithms are provided in Chapter 2.

The goal of P2P live video streaming is to deliver high quality video to each peer in real time such as an ongoing sport game. However, due to the dynamics of peers and the inefficient structure of the network, current P2P LVS software causes problems such as long start-up delay and non-smooth playback for end users [5]. Next, we will address these problems in detail.

\subsection{Research Problems}

Although the current existing P2P LVS software supports a large variety of live channel selection, end users have experienced problems such as non-smooth playback during the time watching live content. However, nowadays, the network downloading bandwidth is large enough for multimedia content, even for high-definition video content. Therefore, downloading bandwidth is not considered as the bottleneck of performance. Instead, the problems can occur for many other reasons. Below, we discuss two of these reasons in detail.

1. The first reason is low overall throughput. Here, the overall throughput means the average speed (bytes/sec) that one peer can download the live video. According to the current architecture of P2P network, users usually download data simultaneously from different users. The overall throughput is the sum of the downloading speed of each data 
stream. However, for P2P LVS, due to the lack of future content, only a few users can upload video content for use by all other users. As a result, the overall throughput for each user is low due to the limited uploading bandwidth of video content. If the overall throughput is less than the video play back rate, then there will be long start-up delay or non-smooth playing back when live content is played.

2. The second reason is low sequentiality. For P2P LVS, the video streaming is divided into chunks ( 8 to $256 \mathrm{~KB}$ ). Here, the sequentiality means the percentage of chunks that are downloaded sequentially. The chunks must be sequential, i.e. there is no missing chunk between any two chunks, in order to be played. In this case, if the downloading mechanism results in low sequentiality, for example, downloading new video chunks ahead of old video chunks, then the video can't be played smoothly no matter how high the overall throughput is achieved.

The above discussion shows that we need to have both high throughput and high sequentiality to achieve high performance for end users. However, one study [6] suggests that throughput and sequentiality are in a trade-off relationship, which means that higher throughput will result in lower sequentiality, and vice-versa. As a result, there will be two extreme situations when one of the factors is maximized.

1. When overall throughput is high and sequentiality is low, it means that the downloading speed is high. However, the video can't be played because the chunks are not in order. In this case, the video can't be played until a group of chunks being played are downloaded in order. 
2. When overall throughput is low and sequentiality is high, it means that most of the downloaded chunks are in order, but the download speed is low. In this case, the video can be played, but users will experience many pauses due to low downloading speed.

Therefore, a mechanism is needed to balance the throughput and sequentiality in order to optimize the overall performance. Previous researches introduced different architectures for building P2P networks for video streaming applications, such as the path-driven algorithms [7-15] and data-driven algorithms [16-29]. However, these researches didn't consider the physical locations of peers when building the P2P network. Besides, most of researches apply the combination of Rarest-First and Naive Sequential as the video chunk selection algorithm, which still result in low sequential throughput due to the weaknesses of these two algorithms. To our best knowledge, there is no such study that can improve the overall performance for P2P LVS application as well as localize the traffic as much as possible.

\subsection{Research Objective}

Based on the discussion above, the objective of our research is to design a P2P architecture for organizing peers for content distribution of live streaming, and to design an algorithm for optimizing data exchange among those peers, in order to improve both throughput and sequentiality and finally achieve smooth play-back for P2P LVS.

\subsection{Our Approach}

By carefully analyzing the advantages and disadvantages of existing systems and the behaviour of users during a P2P live streaming session, we design a multibit Trie 
structure called Bottom-Up Trie (BU-Trie) for P2P live streaming. The main design goals of BU-Trie are to minimize the start-up delay, localize the traffic, and improve the playback continuity by increasing the sequential throughput. Different from traditional tree-based overlay, BU-Trie has two phases: diffusion phase and swarming phase. The diffusion phase is designed for fast distribution of random video chunks to local content network. At the same time, the swarming phase is designed to optimize data exchange within each local content network. The diffusion phase is where peers form the BU-Trie structure and source node pushes the streaming data to certain peers. The swarming phase is where the peers download streaming data from each other.

We also use Opnet [31] as the simulation tool and develop several comparison tests to evaluate the performance of our BU-Trie design. We compare the simulation results of BU-Trie with other existing algorithms to show the advantages of BU-Trie, as well as verify our approach.

\subsection{Summary of Contributions}

The main contributions of the thesis are as follows:

1. Developed a Multibit trie structure for P2P LVS that builds the trie inversely from leaf nodes back to root node. This design minimizes the initial end-to-end delay for distributing live video streaming from source to end users by discovering the physical locations of the peers and connecting peers who are close to each others in terms of physical locations. 
2. Designed a swarming phase based on the characteristics of P2P LVS applications. The swarming applies the Most Popular Chunk First (MPCF) algorithm as the chunks selection strategy. The proposed MPCF algorithm improves the system performance by achieving higher sequential throughput comparing to existing algorithms. The gain in throughput would result in better playback continuity and shorter start-up delay. Also developed a mathematical model to prove the advantage of MPCF design.

3. The simulation results show that the end-to-end delay of distributing video stream from a source to the end users has been decreased. Besides, the sequential throughput of downloading video chunks has been increased as expected. Several comparison tests also show that BU-Trie design with MPCF algorithm improves the existing chunk selection algorithms in terms of throughput and sequentiality. The simulation results also match our analytical results, which prove our mathematical model is correct and acceptable.

\subsection{Thesis Organization}

The remaining chapters of this thesis are organized as follows:

Chapter 2: Introduce the background knowledge for P2P live video streaming. Survey the literature on the current P2P live video streaming architectures.

Chapter 3: Introduce the overall structure and the building process of BU-Trie.

Chapter 4: Introduce the detailed design of diffusion and swarming phase of BU-Trie. A chunk selection algorithm (MPCF) is proposed to improve the overall performance. 
Chapter 5: Introduce the implementation of BU-Trie in OPNET and collect the results from the simulation. Also, compare the simulation results with the existing approaches.

Chapter 6: Present the conclusion and recommendations for future research.

\subsection{Publication}

B. Zhang, C.Huang, J.Yan, "Bottom-Up Trie Structure for P2P Live Streaming," IEEE International Conference on Communications (IEEE ICC 2012), Ottawa Canada, June 2012 


\section{Chapter 2}

\section{Background Knowledge}

This chapter provides the background information for the material presented in this thesis. It reviews Peer-to-Peer (P2P) networks and surveys existing related works on P2P live video streaming. First, a survey of different types of existing P2P live video streaming (LVS) systems in terms of architecture is introduced. This survey focuses on the structures of building P2P LVS systems, for example, how to discover and organize peers, which is related to our diffusion phase design of the BU-Trie. Some popular algorithms are introduced and compared. Furthermore, a discussion of different types of downloading algorithms for P2P LVS system is also provided. This discussion focuses on the existing video chunk selection and downloading strategies, which is related to our swarming phase design of the BU-Trie. We show some popular chunk selection strategies and compare their advantages and disadvantages.

\subsection{Review of Peer-to-Peer Live Video Streaming (P2P LVS)}

In this section, we will provide an introduction to P2P LVS, including the different architectures of P2P LVS designs, the different video chunk downloading algorithms, and the related works. 
According to the driving element in data distribution, P2P live streaming algorithms can be divided into 3 major categories: path-driven, data-driven, and hybrid-driven.

Most path-driven algorithms are tree-based architectures, which are mainly based on the multicast technique at the application layer. In general, it has high efficiency and low delay for data distribution in a static network. However, the adaptability in dynamic networks is not good enough. Each time before the data transmission, it usually requires building the path which covers all the peers in the network. The actual data distribution will then follow the designed path.

Data-driven algorithm is another typical data distribution mechanism for P2P network. In general, most data-driven algorithms are mesh-based architecture, which have high adaptability in dynamic network. However, it has the weakness of low efficiency and high delay for data distribution. It does not require building the entire path before data transmission. Instead, peers need to exchange status information with each other periodically during the data transmission. By examining and comparing the status of neighbour peers and itself, peer can recognize its demand for certain piece of data and the availability of that desired piece of data on its neighbours. Peer can then pull the data from its neighbours according to certain strategies.

Hybrid algorithm is the combination of path-driven and data-driven algorithm. In general, it has the advantages of both tree-based and mesh-based algorithms, such as high efficiency and low delay in dynamic network. During the data transmission, peers can choose between path-driven and data-driven algorithm depending on the current condition. Usually it starts with data-driven algorithm. During the diffusion process, 
when certain condition is reached, it will partially change to path-driven algorithm. For example, some of the peers start building path for later transmission in order to achieve higher efficiency.

\subsubsection{Path-driven algorithms}

The main key of path-driven algorithm is to design how to choose the path and how to build the path. Different path and structure will affect the system's scalability, efficiency, stability, and delay issue. Nowadays, most of the path-driven algorithms can be divided into 2 categories: single-tree path and multi-tree path.

NICE Protocol [7] is a single tree based path-driven algorithm. In this protocol, peers are represented as cluster mates. Then the cluster mates are grouped together in clusters, which are placed on different layers. One peer in each cluster will be chosen as cluster leader, which will be formed together as a higher layer cluster. The peer in layer $\mathrm{Y}$ is also located in layer $Y-1, \ldots, 0$. The cluster size is between $K$ and $3 K-1$, where $K$ is a constant (e.g. $\mathrm{K}=3$ means the lowest cluster size is 3 and the highest cluster size is 8 ). In this case, there are at most $\log _{\mathrm{k}} \mathrm{N}$ layers, where $\mathrm{N}$ is the number of peers, and the highest layer only contains one peer (root peer). As shown in Figure 1, one of the peers in each bottom cluster is chosen as a leader. For example, A3, B1 and C3 are chosen as the leader of cluster A, B and C, and they are formed together as a Layer 1 cluster called cluster D. B1 is then chosen as the leader of cluster D and promoted to layer 2 cluster. Since B1 is the only node in the highest layer cluster, B1 is the root peer. 


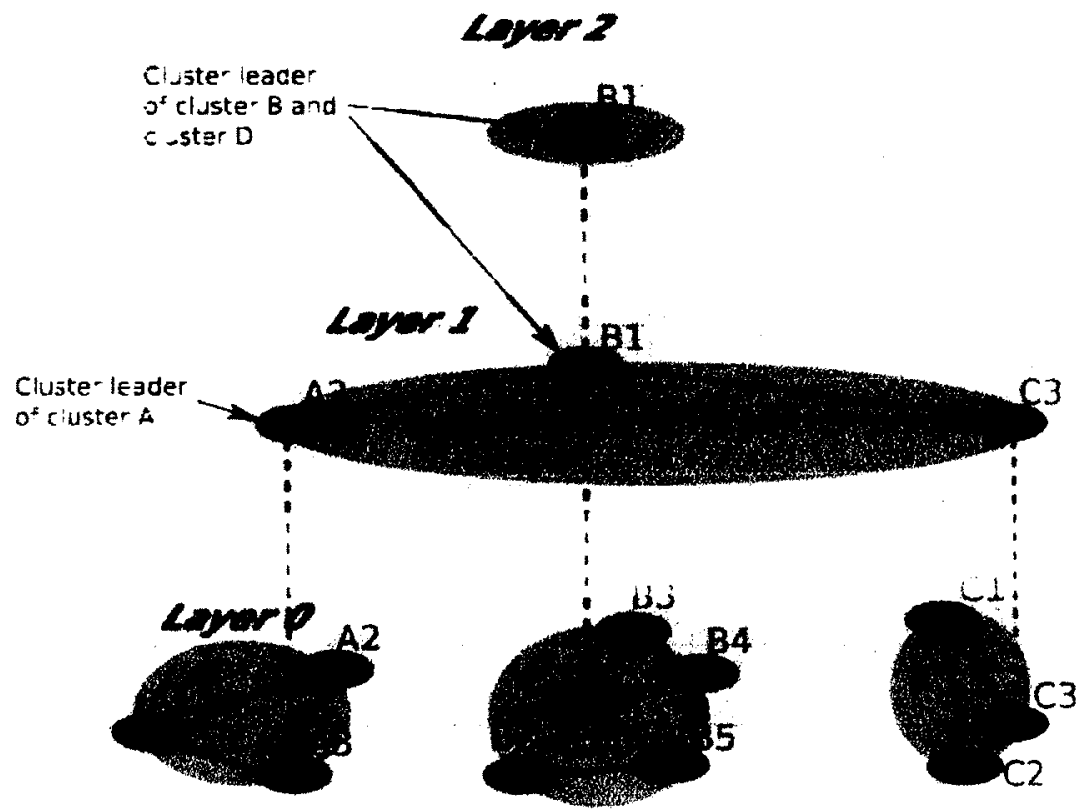

Figure 2.1 Nice Protocol Tree

As shown in Figure 2.1, during the live streaming session, video source will send data to all peers within the same cluster. The leader peer who receives data will then send data to all peers in its cluster. Each leader peer does the same thing until all peers receive the data.

In ZIGZAG [8] is another single tree based path-driven algorithm, there are two topologies: initialization topology and data delivery topology. Initialization topology is shown in Figure 2.2. 


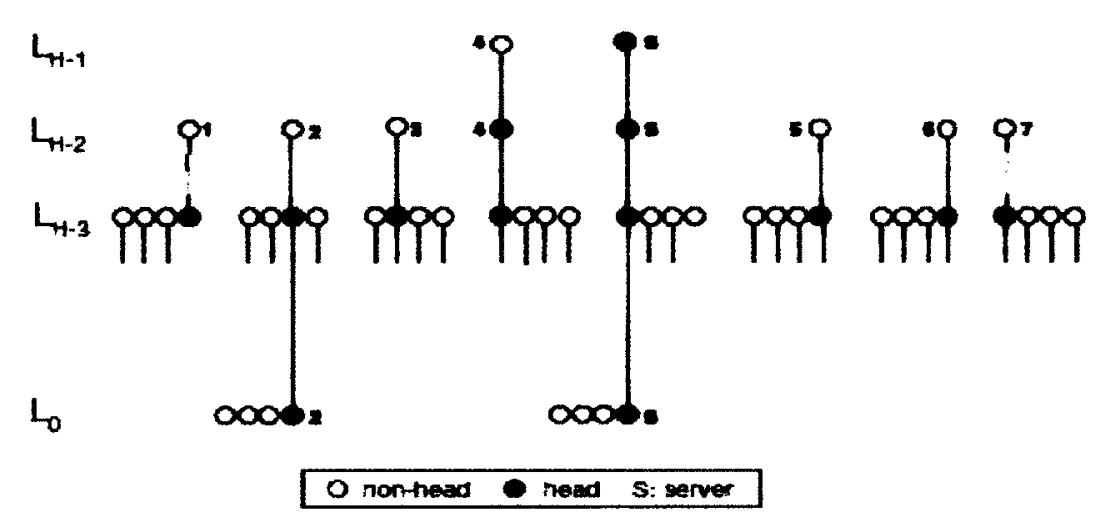

Figure 2.2 Organization of Peers in ZIGZAG

At the beginning, peers are organized in a multi-layer hierarchy of clusters. Layer 0 contains all peers. Basically, if the number of peers at layer $M$ is greater than $3 \mathrm{~K}$, they are partitioned into clusters whose sizes are in $[\mathrm{K}, 3 \mathrm{~K}]$. Otherwise, we reach the highest layer, where peers form only a single cluster. The size of this highest-layer cluster is in [2, 3K]. A layer-Y cluster designates a member peer as its head. The head automatically appears at layer $(\mathrm{Y}+1)$. The cluster partition at layer $(\mathrm{Y}+1)$ is the same as at layer $\mathrm{Y}$. The video source $S$ is the head of any cluster it belongs to. For data delivery topology, however, video stream does not go from the source to head peer as we might think. In a cluster, the head peer has a link to every other non-head peer. The head of a cluster also has a link from one of head peer in other cluster. Figure 2.3 shows the topology for data delivery. Peers can't get data from the head peer within the same cluster. Instead, they get data from head peer in other cluster. The reason of not downloading from local head is because the higher layer head is connected to all lower layer peers. The closer to the source, the larger degree would have. In other words, the bottleneck would occur very early in the delivery path. 


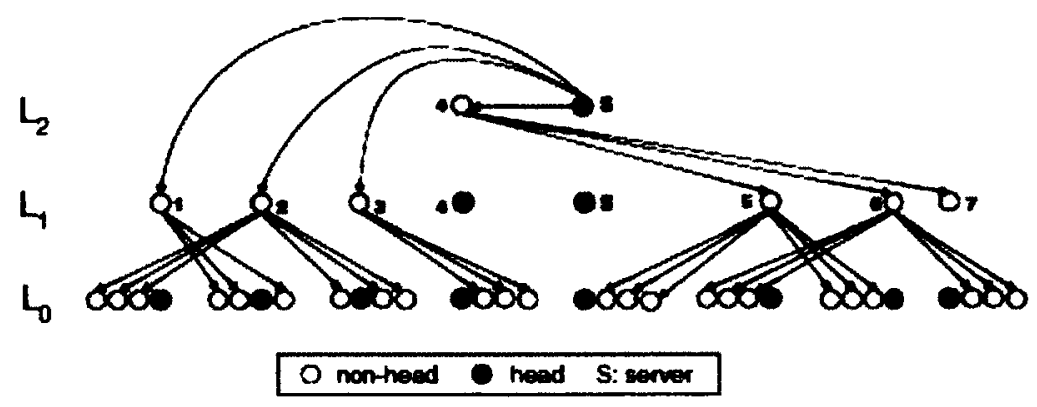

Figure 2.3 Data Delivery Topology of ZIGZAG

The above two algorithm are the most famous two single tree based path-driven algorithms. Path-driven algorithms are the easiest method by applying multicast technique at application layer. It can achieve low delay data distribution without complex video coding technologies, since the path has already been built and peers know where to push the data to or get the data from. The performance is decent for data distribution in static network. However, the adaptability in dynamic network is not good enough. First of all, single point failure has big effect to the system. The time needed for rebuilding tree and recovering is usually long. Secondly, the performance of each peer can introduce large effect to the system. For example, in heterogeneous network, if the tree is built without considering the bandwidth of each peer, then there might be bandwidth bottleneck during the video session when low bandwidth peers are selected as the parent peers in the tree. On the other hand, if the tree is built by considering the bandwidth of each node, then the system is not optimized and delay might be introduced to the system. Thirdly, single-tree based algorithm can lead to unbalance in the system. Peers in higher layer have much heavier load than the leaf peers in lower layers. Finally, the bandwidth 
consumption is inefficient. Leaf nodes can't use the upload bandwidth according to the algorithm.

Based on the disadvantages of single-tree based algorithm, multi-tree based path-driven algorithm is introduced. These algorithms build multiple trees from the source peer to all other peers according to certain strategies. In order to make the system more efficient, the media stream is usually divided into several sub-streams, where each sub-stream is distributed through a tree. Some coding techniques, such as Layered Coding (LC) and Multiple Description Coding (MDC), are usually applied for media stream.

Coopnet [9] is based on multi-path tree topology. Based on this algorithm, the video source firstly uses multiple description coding mechanism on video streaming data to generate several MDC sub-stream. In this method, a media signal is encoded into several separate streams, or descriptions, such that every subnet of them is decodable. Then Coopnet builds multiple distribution trees spanning the source and all the receivers, each tree transmitting a separate description of the media signal. Therefore, a receiver can receive all descriptions. Based on the ability, each peer can choose to join one of the trees or all the tree structure. Depends on which tree the peer joins, each peer will receive the video streaming in different quality. A peer failure only cause its descendant peers to lose a few descriptions. The descendants are still able to continue their service.

Splitstream [14] is similar to Coopnet. It also requires the coding algorithm at the source peer. After generating the MDC sub-stream, a tree is built for each MDC. However, according to Splitstream, each peer can only act as parent node in only one tree it joins. It acts as children nodes in other trees it joins. For example, as shown in Figure 2.4, two 
MDC sub-streams are generated by source. Therefore, there are two trees for the two MDCs. During the video session, each peer can only act as parent peer in one of the tree. For example, peer 2 acts as parent peer in tree 1 , then it can only act as a child peer in tree 2. By doing this, the load is balanced among all peers. The effect of peer churn can also be reduced.

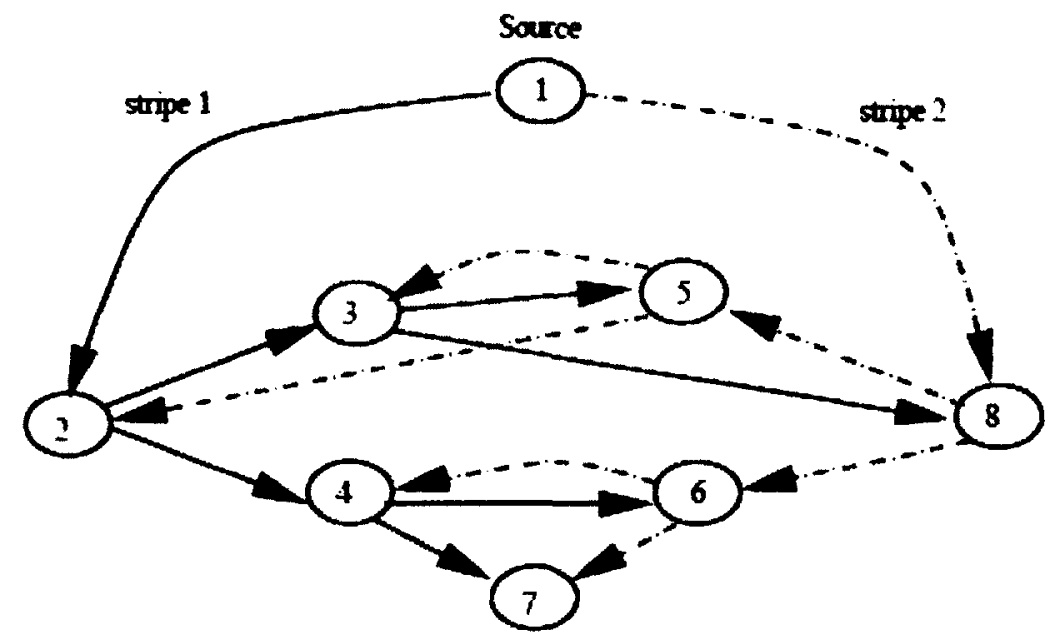

Figure 2.4 MDC Sub-streams of Splitstream

The advantage of multi-tree based path-driven algorithm is that the bandwidth of each peer in the system can be used sufficiently, which balances the whole system. The effect caused by single point failure can also be reduced. However, this type of algorithms is complex and difficult to be implemented in real world.

\subsubsection{Data-driven algorithms}

Different than path driven algorithms, data-driven algorithms focus more on the availability of data. In dynamic network, other than rebuilding tree path, it is more efficient to lead the data streams by their availability. Therefore, during the data 
transmission, data is not distributed through the designed path. Instead, it is transmitted according to the demand of data. In data-driven algorithm, resource scheduling algorithm is the core technique which usually decides the data distribution model. In modern P2P live streaming applications, random pull are usually applied. A peer can build connections with multiple neighbour peers. Neighbour peers exchange bit map message periodically to get the status of data buffer. Peer will firstly recognize the missing data block which is available in neighbour peers. Peer will then send pull request to all randomly selected neighbour peers for that data block. The neighbour peer will then send the requested data in the request period or the following request period.

Coolstreaming [10] is a data-driven algorithm. The algorithm of how to choose peers is based on the time limitation of the live streaming. A peer firstly gets statistics of which neighbour has certain data piece. After that, it decides which neighbour should be chosen according to time limitation and predicted downloading speed. According to the research paper, the downloading speed is calculated by the bandwidth, however it doesn't give how to find out the bandwidth between two peers. Measuring the bandwidth for dynamic application is a very difficult job. Also, the cost to maintain information for each piece of data is very large. As a result, it might generate large delay as the video session goes on.

DONet [11] is based on Gossip protocol. The main idea here is that each peer exchanges information of a certain piece of data with other neighbour peers periodically. For each segment of a video stream, a DONet node can be either a receiver or a supplier, or both, depending dynamically on this segment's availability information, which is periodically exchanged between the node and its partners. An exception is the source node, which is always a supplier. Each DONet node has a unique identifier, such as its IP address, and 
maintains a membership cache (mCache) containing a partial list of the identifiers for the active nodes in the DONet. In a basic node joining algorithm, a newly joined node first contacts the source node, which randomly selects a node from its mCache and redirects the new node to that node. The new node can then obtain a list of partner candidates from the deputy, and contacts these candidates to establish its partners in the overlay.

The above two algorithm are famous mesh based data-driven algorithms. In general, for a mesh-based data-driven algorithm, the video streaming is usually divided into small chunks. Depending on the chunk selection strategy applied, the missing video chunks are pulled by one peer from its neighbour peers who have already had the desired data. Most of the time, the mesh-based structure is robust to the unpredictable peers' behaviours since each peer always maintains a list of its neighbour peers and has multiple neighbour peers to download video chunks from. However, mesh-based overlay has relatively poor performance for live contents due to the limited availability of future contents and the poor sequentiality of the received video chunks, which may result in non-smooth playback and long waiting time.

\subsubsection{Hybrid Algorithms}

Hybrid algorithms are the mixed strategies of path-driven and data driven algorithms. In general, they have the advantages of both tree-based and mesh-based algorithms, for example, hybrid algorithm may achieve high adaptability and high efficiency at the same time.

CliqueStream [32] is one of the famous hybrid algorithms in recent years. In [32] the authors propose a clustered P2P overlay network called CliqueStream to achieve both 
locality and robustness. In this algorithm, the peers are organized into several clusters which have their own identifiers. One or more stable peers in each cluster will be selected and assigned relaying role. Then a content delivery tree is constructed out of the stable peers and the content is pushed through the content tree. On the other hand, the unstable peers in each cluster will then create a mesh around the stable peers and pull the content. By doing this, CliqueStream can achieve both the high efficiency of content delivery over the tree structure overlays and the high robustness over the data-driven mesh overlays.

Since the chunk selection strategy also plays an important role for P2P LVS applications, we will show some related works about chunk selection and downloading in next section.

\subsection{Chunk Selection Strategy}

Chunk selection strategies are usually applied to mesh-based overlay network in order to improve the performance. In this section, we introduce some existing chunk selection and downloading strategies, as well as some related researches.

\subsubsection{Chunk Selection Algorithms}

There are two most popular algorithms that are widely used in P2P streaming types of applications: Rarest-First and Naive Sequential [33]. Rarest-First is where peers always download the video chunks that are shared by the least number of peers. As a result, video chunks are evenly distributed among the network. Naive Sequential is where peers download all video chunks in order. For example, peers always download the first missing chunk. By applying Naive Sequential, all the video chunks can be played as soon as they are downloaded. Previous research [33, 34] claimed that Rarest-First can achieve 
the highest continuity for video playback by maximizing P2P sharing. However, it will result in high start-up latency, since the video chunks downloaded at beginning are usually not in order and therefore cannot be played. Also this algorithm tends to favor latest chunks. This may lead to old chunks getting starved. On the other hand, Naive Sequential can achieve low start-up latency, since all downloaded chunks are in order and can be played immediately. However, Naive Sequential fails to maximize P2P sharing, which makes overall throughput for each peer to be low. As a result, it has poor playback continuity.

In [6], the authors claimed that throughput, robustness, and sequentiality are the three most important attributes that determine the performance of $\mathrm{P} 2 \mathrm{P}$ video streaming. Throughput measures the average completion time for peers to download the entire file. Higher throughput will result in faster downloading speed. Robustness measures the ability to withstand heterogeneous network conditions such as churn. Sequentiality measures the degree to which the file is retrieved sequentially. Based on some assumptions, the author uses mathematical model to show that there is a fundamental tradeoff that exists between these three attributes. For example, we can't maximize throughput and sequentiality at the same time. The authors showed three methods to balance the TRS tradeoff, however there is no optimum solution provided.

Paper [33] [34] analyzed chunk selection strategy for downloading video stream. In [33], the authors showed two simple strategies of downloading video stream chunks: Rarest First and Greedy. Rarest First is a well-known strategy for P2P file sharing system, where peers always download the data chunk that are shared by the least number of peers. By applying Rarest First, video chunks are evenly distributed among the network. Greedy is 
where peers download all video chunks in order. For example, peers always download the first missing chunk. By applying Greedy, all the video chunks can be played as soon as they are downloaded. The authors claimed that Rarest First can achieve the highest continuity for video playback by maximizing P2P sharing. However, it will result high start-up latency, since the video chunks downloaded at beginning are usually not in order and therefore can't be played. On the other hand, Greedy can achieve low start-up latency, since all downloaded chunks are in order and can be played immediately. However, Greedy fails to maximize P2P sharing, which makes overall throughput for each peer to be low. As a result, Greedy has poor continuity.

The authors then propose a mixed strategy, in which both Rarest First and Greedy are applied. In that strategy, the buffer space is divided into two parts. The first part of buffer space is applied by Rarest First algorithm. Greedy is then applied to the second part of the buffer map. By adjusting the value of $m$, the maximum continuity can be achieved. For the same value of $\mathrm{m}$, the start-up latency is also relatively low. The authors then tested the optimum solution for small and large scale network. The results show that this mixed strategy is steady and acceptable.

In [34], the authors used similar idea to analyze the problem. Instead of considering the start-up latency, the objective is to maximizing playback continuity. The authors proposed to use density dependent jump Markov process (DDJMP) to analyze the chunk selection policy, and proved that the DDJMP is asymptotically exact when scaling up the system. A segmentation algorithm is also proposed to reduce the computational complexity of evaluating the performance of a chunk selection policy. This algorithm demonstrates that the more the server is able to upload, the more the peers can afford to 
use the local greedy policy. The authors then showed that the optimal chunk selection policy has the "V" shaped structure, and present a distributed adaptive algorithm so that the server can notify all peers about this optimal chunk selection policy whenever there is any change in the system parameters.

\subsubsection{Related Researches}

Several other researches [35-40] also aim to improve the throughput of downloading video chunks. In [35], an optimal routing solution is provided to maximize throughput under the fairness constraint in layered P2P streaming. The paper used multi-commodity flow theory to achieve intra-layer and inter-layer fairness in the receiver heterogeneity in P2P multimedia distribution. In [36], the authors proposed a networking and video coding co-design for another overlay P2P-based IPTV system. A graph construction mechanism is developed which reduces disconnect time and isolated peers. The authors presented experimental results showing the mechanism is able to deliver optimal video quality in dynamic networking environments. Many other researches [41-46] are proposed to improve the overall performance of P2P LVS as well. 


\section{Chapter 3}

\section{Overall Structure of BU-Trie}

In this chapter, we define the overall structure of Bottom-Up Trie. We show the detailed design of BU-Trie. Firstly, we introduce the method we use to determine physical location of peers. Then we show how to build the BU-Trie from leaf nodes to parent nodes step by step. Besides, we will also introduce how to organize peers for certain live content.

\subsection{BU-Trie Setting Up}

As mentioned, Bottom-Up Trie is a multi-bit Trie structure designed for P2P live streaming in order to improve the overall performance. In this section, we show the detailed design of Bottom-Up Trie and how to build it inversely from leaf nodes to root node step by step.

The main idea of BU-Trie is to let each node in the network download data from its closest neighbour nodes. We use the term "closest neighbour nodes" to refer to the nodes that are geographically close to each other. In order to determine whether the locations of two nodes are geographically close to each other, we compare the IP addresses of the two nodes. According to [47], most of the IP address allocations are related to nodes' physical 
locations, and the physical locations of IP prefix rarely changes. In this case, if the IP prefixes of two nodes are the same, for example, the higher 24 bits in IP addresses are the same, then we consider that these two nodes are geographically close to each other. Or otherwise, even though the physical locations of two nodes with the same IP prefix are not close to each other, they are usually in the same network domain or under the same ISP, where closeness means the cost is cheaper because they are in the same ISP.

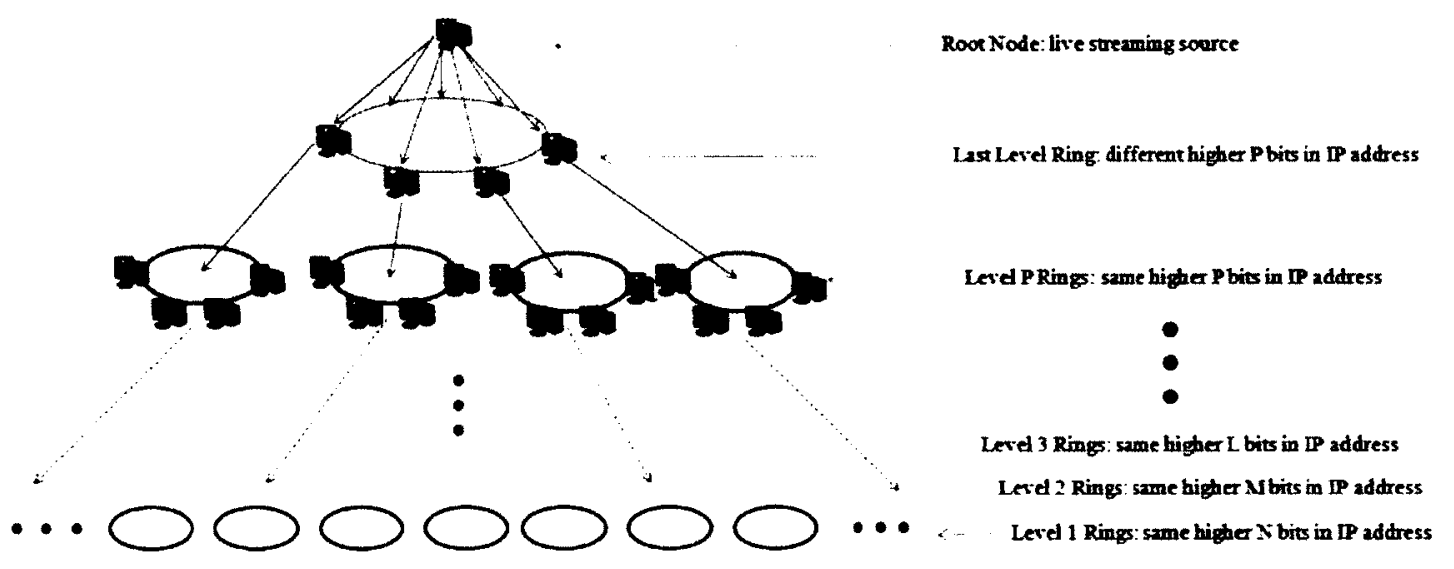

Figure 3.1 Bottom-Up Trie Architecture

By using the philosophy described above, we can break down all peers and organize them into a hierarchy of small groups, where all peers in the same group are close to each other. We assume there is a registration system for a P2P application. The registration system can be either centralized or distributed.

To start with, each peer contacts the registration server with its IP address. The server will compare all peers' IP addresses and divide the peers into different groups according to the higher $\mathrm{H}$ bits in their IP addresses. Note that the peers in the same group will have 
the same higher $\mathrm{H}$ bits in their IP addresses. In other words, only the last $32-\mathrm{H}$ bits in their IP addresses are different for the peers in the same group. As shown in Figure 3.1, each circle represents a group. At level 1, all peers in the same circle (or group) have the same higher $\mathrm{H}$ bits of their IP addresses where $\mathrm{H}$ is set to be 28 as an example. Therefore, there are maximum 16 different peers within the same Level 1 group after the first division. It is easy to see that, based on our definition of closeness, the peers within the same group are close to each other.

After step one, each peer will be informed of its neighbour information by the registration system. The peers in the same group will form a DHT (Distributed Hash Table) ring with fingers if necessary. Each peer will also be assigned an identifier based on its last $32-\mathrm{H}$ bits of IP address.

The third step is to choose a parent peer in each group for a P2P application. We assign a key value to each live streaming source. The key value is then hashed to a node identifier. In each Level 1 group, we select the node with closest node identifier in the last $32-\mathrm{H}$ bits as the parent for the specific application. For example, if the last 4 bits of the hash value of a video session is 0101 , then the nodes with the same last 4 bits of IP addresses in each group will be chosen as the parent node in this group for this specific video session. If no node has the same last 4 bits of IP address equal to 0101 , then the next closest node in terms of IP address will be chosen. The host node will serve as a parent in the Level 1 group. It should be noted that different applications will select different parent nodes. This will allow loads to be distributed relatively evenly among different peer nodes.

In step 4, the registration system selects a value $M$ as the number of bits to compare for Level 2 in the hierarchy, where $\mathrm{M}<\mathrm{H}$. The Level 1 groups with the same first $\mathrm{M}$ bits in 
their IP addresses will be in the same Level 2 group. There will be maximum H-M Level 1 groups in a Level 2 group. The parent node in each Level 1 group for each application will serve as the representative for the Level 1 group in Level 2.

In step 5, the registration system will inform each parent of Level 1 its neighbours in Level 2. These parent nodes then form a Level 2 DHT ring with fingers if necessary. A node identifier based on the bits $\mathrm{N}$ to $\mathrm{M}$ will be assigned to each Level 1 parent as their Level 2 identifier. For each application, the node with closest Level 2 identifier for the bits $\mathrm{N}$ to $\mathrm{M}$ of the hash of the specific application key value will be selected as the parent of Level 2 nodes.

For example, Level 1 parent nodes with the same higher 24 bits of IP addresses can be organized to form the Level 2 group. Then for the hashed key value of the same live streaming source, the most matching node in each Level 2 group, for example the node has the same bits 25 to 28 or the node with closest bits 25 to 28 , will be chosen as Level 2 parent in the level two group. Up to this point, the second bottom level of the BU-Trie is finished. During the live streaming transmission later on, the level two parent node will push data to its level one parent nodes, and then the level one parent nodes will further push the data to all leaf nodes connecting with.

We use the same strategy to recursively build the third level, the fourth level, etc, until the source of the application is selected as the last level. Here we assume that the key of the live content is hashed to the IP address of the source. Figure 3.1 shows the overall architecture, where $\mathrm{P}<\ldots<\mathrm{L}<\mathrm{M}<\mathrm{H}<32$. It is easy to see that the overall structure forms a multi-bit Trie. As described above, our unique construction process starts from the 
bottom level and moves level by level to the root node. Therefore it is called a BU-Trie (Bottom-Up Trie) structure.

After building the BU-Trie, each node has the information of its parent node and child nodes. When live streaming session starts, each node tries to contact its parent node in the same group. If the parent node has the contents, it will send the contents to the node directly or direct the node to contact a peer node in the same group which happens to have the requested contents. Otherwise the parent node will contact its parent at the next level. This process will continue until the root node is reached. The video contents are therefore broadcasted from the root node to the whole Trie.

By using the BU-Trie structure, each new peer can easily determine its position in the Trie for certain live streaming, and it can easily choose the closest peer to get the desired data as well. This will minimize the delay for content delivery. If a leaf node happens to leave, only the DHT table in the lowest level group the leaf node belongs to needs to be updated. On the other hand, if a parent node at Level $x$ leaves a session, another node within the same group in Level $x$ will be selected immediately as the parent. Related DHT labels in the level $x+l$ to the lowest level will be updated. These DHT tables are the closest ones to the leaving node and therefore can be updated very fast. In general, the BU-Trie is robust to the unpredictable peers' behaviours since it can be recovered easily following simple rules.

The BU-Trie structure also maintains the benefits of DHT table by providing alternatives for peer selection within the same group. The DHTs in a BU-Trie are constructed into a hierarchy, which makes it more scalable than mesh overlays. It also achieves our goal to localize traffic in lower levels of the trie as much as possible and henceforth minimize 
delay. Each application session has its own BU-Trie. This will allow peers to share load across different live sessions and avoid bottleneck problem.

\subsection{Two Phases Design Approach}

After building the BU-Trie structure, the next step is to design how to distribute the live content. Based on the discussion in Chapter 2, the main design goals of BU-Trie are to minimize the start-up delay, localize the traffic, and improve the playback continuity by increasing the sequential throughput. After carefully analyzing the advantages and disadvantages of different P2P LVS structure, chunk selection strategies and the behaviour of users during a P2P live streaming session, we decided to achieve our design goals by having two phases: the diffusion phase and the swarming phase. The diffusion phase is designed for fast distribution of random video chunks to local mesh network. At the same time, the swarming phase is designed to optimize data exchange within each local DHT network. The diffusion phase is where peers form the BU-Trie structure and source node pushes the streaming data to certain peers. The swarming phase is where the peers download streaming data from each other.

The purpose of the diffusion phase is to group the local peers together and design the path from the video source to all groups. By doing this, the source node can distribute streaming data as widely as possible to each local domain. Different from other treebased architecture, the BU-Trie is formed and built inversely from leaf nodes (or child nodes) back to the root node (or parent nodes). Peers in the same level of the Trie also form a ring structure by applying Distributed Hash Table (DHT) [30] building process 
according to their IP addresses. As mentioned above, the main design goal of diffusion phase is to minimize the initial end-to-end delay for distributing live contents from the source to end users by discovering the physical locations of the peers and connecting peers who are close to each others in terms of physical locations. According to our algorithm, a new peer who just joins the network will be assigned to one of the local DHT rings according to its IP address. For each live streaming session, a BU-Trie is formed by all the peers who are willing to download the live content before the actual data transmission. The BU-Trie is dynamic and will adjust its structure according to existing peers' absence and new peers' presence. We show that by forming a BU-Trie, the live content can be distributed as widely as possible to users in different domains.

The swarming phase of the BU-Trie starts in parallel to the diffusion phase. The swarming phase is designed to optimize data exchange within each local DHT network. We show that by applying the proposed Most Popular Chunk First (MPCF) algorithm, the sequential throughput of downloaded chunks is increased compared to other approaches. The gain in throughput would result in better playback continuity and shorter start-up delay.

The following Chapter 4 will introduce the diffusion phase and the swarming phase design in detail. We also propose an algorithm called Most Popular Chunk First (MPCF) to work with the swarming phase design. 


\section{Chapter 4}

\section{Two Phases of BU-Trie}

In this chapter, we introduce the diffusion phase and the swarming phase of Bottom-Up Trie. The diffusion phase and swarming phase of the BU-Trie start in parallel after the video session started. Firstly, we show the detailed design of diffusion phase. Then we will show objective of the swarming phase design and why we need this second phase. Next, we propose a new algorithm called Most Popular Chunk First (MPCF) for video chunk selection. The MPCF is the core algorithm of swarming phase, which decides the order of chunks downloading. We also compare the MPCF algorithm with some existing chunk selection algorithms to show the advantages of our approach. We then develop a mathematical model for estimating the sequentiality. At the end of this chapter, we describe how the MPCF algorithm can be implemented with DHT tables and bitmaps.

\subsection{Diffusion Phase of BU-Trie}

In this section, we introduce the diffusion phase of BU-Trie. As discussed in previous chapters, the purpose of diffusion phase is to achieve fast distribution of random video chunks to local content network, in order for local peers to exchange the missing chunks with each other as early as possible. Figure 4.1 shows the video chunks distribution in diffusion phase. 


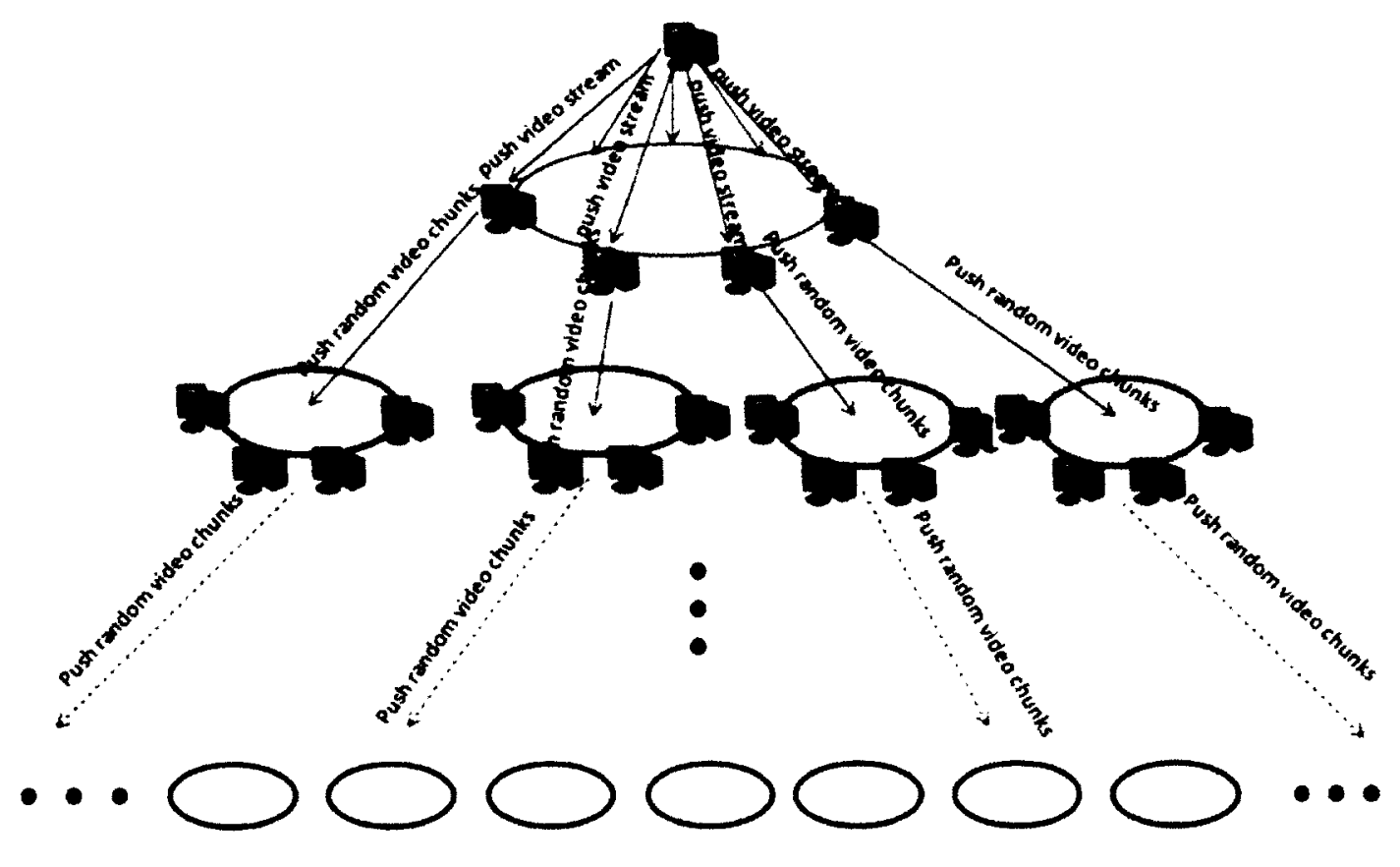

Figure 4.1 Diffusion Phase

The diffusion phase starts right after building the BU-Trie. During the diffusion phase, the root node continuously push video stream to each of the next level parent nodes. Then these parent nodes will further push the stream to their child nodes. This process will continue until level-1 parent nodes are reached. Since the uploading bandwidth is limited, a level-1 parent node will randomly push video chunks to different peers in the same group. As a result, each peer as a leaf node may receive random number of video chunks. Figure 4.2 shows an example of two peers' bitmap after the diffusion phase. The blocks shown in grey color represent the video chunks that the peer received during diffusion phase so far. The blocks shown in white color represent the missing video chunks. Due to the fact that all video chunks are randomly pushed, these two peers may receive same video chunks or some different video chunks as a result. 

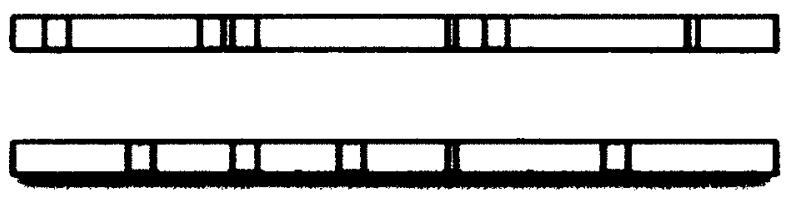

Figure 4.2 Example of Peer's Bitmap after Diffusion Phase

The advantage of this diffusion phase is that video stream can be fast distributed from the root node to a wide area covered by the Trie. Even though there may be many missing chunks at this moment, the speed of distribution is high, since the video stream is diffused along the Trie that is built based on closeness.

Once peers start receiving video chunks, the swarming phase starts as well. We will talk about the swarming phase in next chapter. But the basic idea here is that peers in the same local group can exchange and download the missing chunks from each other. For example, as shown in Figure 3.3, most of the video chunks received by these two peers are different. In this case during the time of waiting for new chunks to be pushed, the two peers can actually exchange the video chunks with each other. This exchanging of video chunks will increase the overall efficiency of the network.

The diffusion phase will continue as the root node generates new live video stream, and it will not stop until the live video session ends. In conclusion, by applying the diffusion phase, random video chunks can be distributed fast to all end users. The delay time of distributing video chunks is predicted to be very low, since peers always get video chunks from the neighbour peers who are physically close to them. In Chapter 5 , we will use simulations to demonstrate that the end-to-end delay of distributing video chunks is reduced dramatically by applying the diffusion phase as predicted. 
As discussed above, the swarming phase starts in parallel with the diffusion phase. Now we will introduce the swarming phase in next section.

\subsection{Objective of Swarming Phase}

The swarming phase of BU-Trie design is the second phase that starts in parallel with the diffusion phase. In previous chapter, we discuss that the source node can't distribute the entire video stream to all end users due to the limit of uploading bandwidth. As a result, end users will only receive partial video streaming, e.g. video chunks, during the diffusion phase. If we consider the entire diffusion phase as a push-driven process that distributes video chunks to selective peers in a wide area, then the swarming phase is a pull process that allows peers in a local area to exchange and download the video chunks that are missing as shown in Figure 4.3.

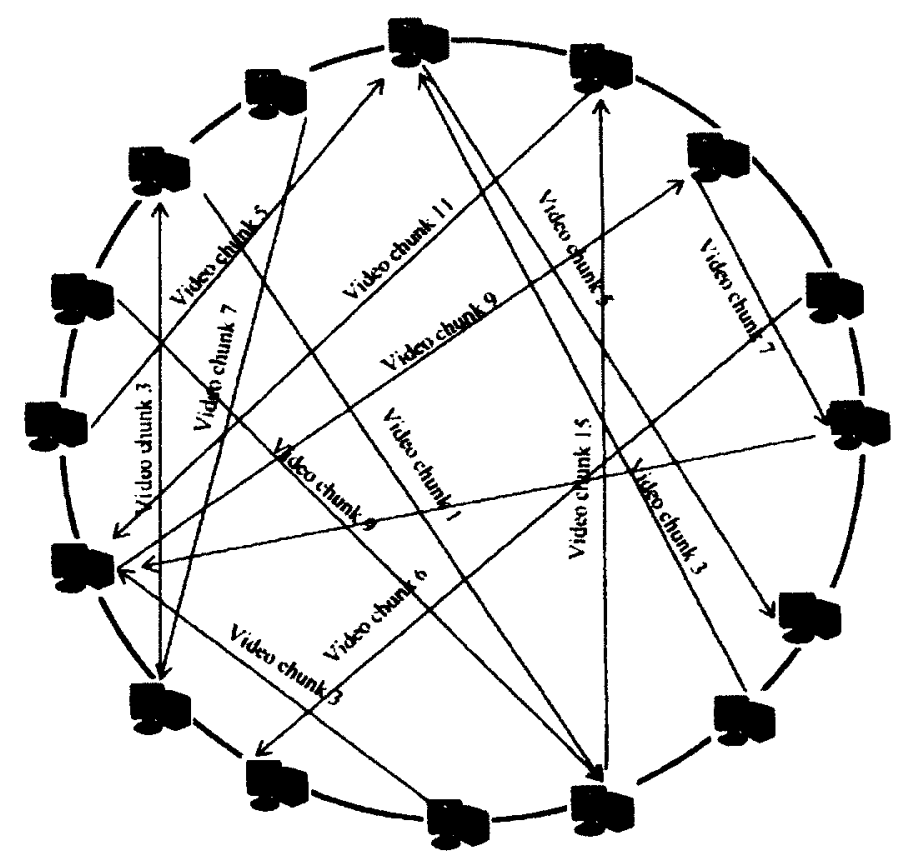

Figure 4.3 Example of Downloading Missing Chunks in Swarming Phase 
The question here is where to download the missing chunks and which chunks need to be downloaded firstly. Therefore, the objective of our swarming phase is to design an algorithm of exchanging/downloading video stream chunks between peers, which optimize the performance of P2P live video streaming.

As discussed in Chapter 2, the performance of a P2P system can be measured by three metrics, which are throughput, sequentiality, and robustness. However robustness is less important for P2P live video streaming. So we will focus on the first two metrics in this paper. To be more specific, these two attributes in our swarming phase design are defined as the following:

1. Throughput is defined as the speed that one peer can download the video chunks, for example, the number of bytes downloaded per second. The higher throughput means the more video chunks can be downloaded each second. In our swarming phase design, we focus on the average value of the throughput. For example, one user has downloaded 36,000 video chunks in 1 hour, then the average throughput is equal to 10 chunks/sec.

2. Sequentiality is defined as the percentage of chunks that are downloaded sequentially. We call the chunks are downloaded sequentially if the order of downloading chunks is same as the order of generating chunks by the source node. For example, one user has downloaded 100 video chunks. If there is no missing chunk from chunk 1 to chunk 30 in terms of time scale, and the chunk 31 is missing, then the sequentiality is equal to $30 \%$.

Ideally, we want both two metrics to reach the maximum values. However, based on the mathematical analysis provided in [6], there is a fundamental trade-off between these two metrics. In other words, the two metrics can't be maximized at the same time. Therefore, 
our goal is to design a method that can reach relatively high throughput and sequentiality, which improves the performance of P2P live streaming.

Moreover, end to end delay (ETE delay) of packet and number of inter-domain packet are another two metrics we will focus on. ETE delay of packet is measured by the waiting time from the time of sending one chunk by the source node to the time of receiving this chunk by a leaf node, which directly affect the initial buffering time of the live streaming. We will also try to reduce the number of inter-domain packets during the live streaming session, which benefits carriers economically.

\subsection{Swarming Phase Design and MPCF Algorithm}

In this section, we show the detail design of the swarming phase. The algorithm we proposed for the swarming phase is called Most Popular Chunk First (MPCF). We firstly define the MPCF algorithm and show how does it different from other existing algorithms. Then we show how to apply the MPCF algorithm to the swarming phase step by step. We show a simple example of how to selection missing chunk by MPCF algorithm in the swarming phase.

\subsubsection{MPCF Algorithm}

As mentioned in Chapter 2, there are two most popular algorithms that are widely used in P2P streaming types of applications: Rarest-First and Naive Sequential. 


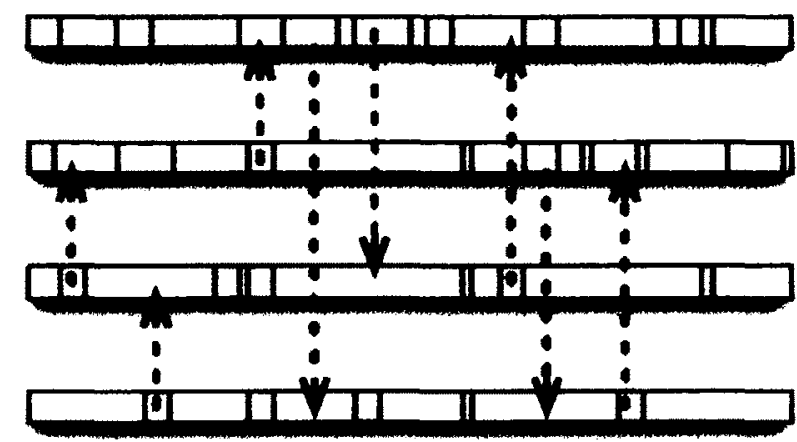

Figure 4.4 Rarest-First Chunks Selection Algorithm

Rarest-First algorithm is shown in Figure 4.4. This algorithm has been widely used in P2P file sharing system. In this algorithm, each peer maintains bitmaps of all other peers indicating which chunks are available, then downloads the rarest-shared chunks from the available peers. By doing this, the system optimizes the even distribution of all chunks. [6] uses mathematical model to show that Rarest-First algorithm can achieve maximum throughput and robustness. This is because this algorithm maximizes the number of sources for each chunk in the system, so both of the throughput and Robustness increase as the number of available sources increases. However, the mathematical model also proves that Rarest-First algorithm achieves very low sequentiality. This is because the rarest-shared chunks are always the chunks that were generated lately in terms of time scale. This algorithm forces peers to download the later generated chunks before earlier generated chunks. Therefore, the sequentiality is very low based on our definition in previous section. As a result, the Rarest-First algorithm is not good for live streaming applications, since the downloaded chunks are not in sequence and therefore can't be played, although the average downloading speed is high. 


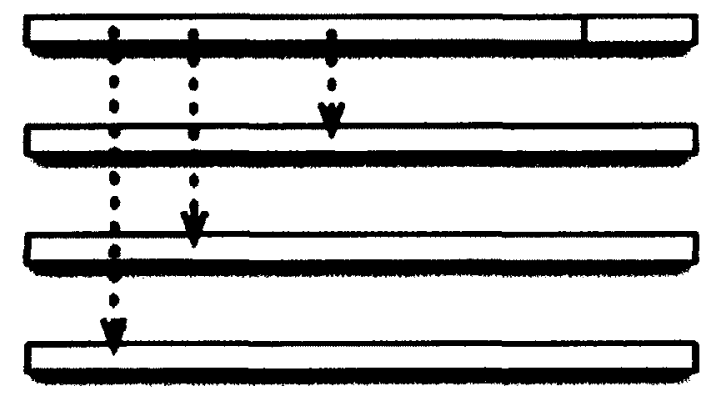

Figure 4.5 Naive Sequential Chunks Selection Algorithm

Naive Sequential algorithm is shown in Figure 4.5. In this algorithm, peers always download the chunks in the order that those chunks are generated and would be played back. In other words, peers always download the first missing chunk in terms of time scale. By doing this, all chunks downloaded are useful and can be played back. [6] uses mathematical model to show that Naive Sequential algorithm can achieve maximum sequentiality, which is equal to 1 . This is because all downloaded chunks are in sequence. However, the mathematical model also proves that Naive Sequential algorithm achieves low average throughput. This is because the chunk distribution is heavily biased toward early chunks. For example, almost all peers have the first chunk and only the source node has the last chunk (we assume peers depart as soon as they obtain the last chunk). The downloading speed for later chunks is low. Besides, this algorithm is too strict in terms of downloading order. If the first missing chunk is not available, the peer can't download any other chunk but just wait.

Both of these two algorithms have their own advantages, but they are not perfect for P2P live streaming according to our discussion. By considering the characteristics of $\mathrm{P} 2 \mathrm{P}$ live streaming, we propose an algorithm called Most Popular Chunk First (MPCF). 
In MPCF algorithm, each peer maintains bitmaps of all other peers in the same local group indicating which chunks are available. However, contrary to Rarest-First algorithm, MPCF lets peers download the most popular chunk first, i.e., always download the chunk that is shared by the most number of peers. If the most popular chunk is unavailable, for example due to peer churn or limited node capacity, then the next most popular chunk will be selected and downloaded. Figure 4.6 shows an example of MPCF algorithm.

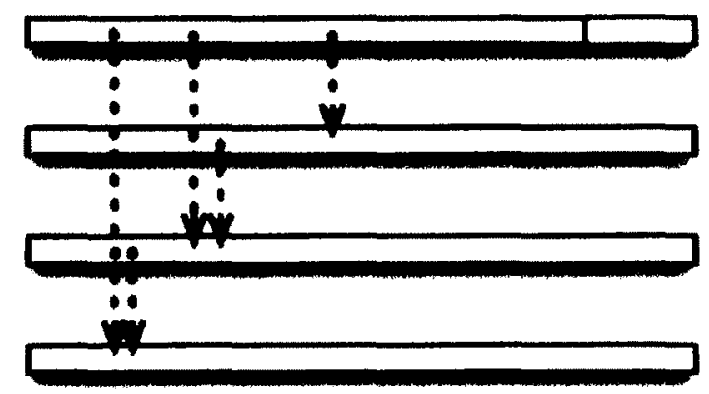

Figure 4.6 Most Popular Chunk First (MPCF) Chunks Selection Algorithm MPCF is ideal for real-time multimedia applications such as P2P live streaming due to the following reasons:

1. In real-time multimedia applications, the older chunks, the chunks that are generated earlier, are always shared by more peers and therefore are more popular. Based on the definition of MPCF, peers tend to download older chunks ahead of newer chunks. So MPCF can achieve very high sequentiality compared to Rarest-First algorithm which tends to download newer chunks ahead of older chunks. Since the buffer size is limited, older chunks may be obsolete. By downloading oldest chunks first, we avoid the situation that some old chunks are starved in the sense that they have been deleted before fully downloaded. Therefore, with high sequentiality, MPCF is better than Rarest-First algorithm for live video streaming applications. 
2. In Rarest-First algorithm, the rarest-shared chunk always has limited availability due to limited uploading bandwidth. If many peers want to download this rarest-shared chunk simultaneously, there might be longer delay or waiting time due to the limited availability. However, for MPCF, the most popular chunk is shared by the most number of peers, so it has high availability. Even though many peers want to download this chunk simultaneously, the waiting time is short.

3. By comparing with Naive Sequential algorithm, both of them tend to download older chunks ahead of newer chunks (shown in Figure 4.5 and 4.6). However, MPCF is better in the sense that it does not enforce sequential requirement. This allows more flexibility in selecting peers. In some cases, peers running sequential algorithm may need to wait because some chunks may not be available due to limited bandwidth, while peers running MPCF can move on to next most popular chunks that have bandwidth to download. This will maximize bandwidth utilization.

Based on the discussion above, it is reasonable to conclude that MPCF algorithm can achieve higher sequentiality than Rarest-First algorithm and higher throughput than Naive Sequential algorithm, which is ideal for real-time multimedia applications such as P2P live streaming. A simulation test is provided in the next chapter to prove the advantages of MPCF. Next we show how to use the mathematical model to prove that MPCF has high sequentiality as expected.

\subsubsection{Mathematical Model of MPCF}

In this section, we show the mathematical model we build for MPCF algorithm, and prove that MPCF algorithm can achieve high sequentiality. First of all, we define some variables and assumptions for this mathematical model. 
Definition 1. Define $P(x)$ as the probability that a peer will download the xth missing chunk in the next downloading period.

For example, $P(1)=90 \%$ means that the $1^{\text {st }}$ missing chunk has a probability of $90 \%$ to be downloaded in next round. Here, we rank the video chunk according to the timescale of the video. As a result, the $1^{\text {st }}$ missing chunk is the oldest missing chunk according to the time to be generated.

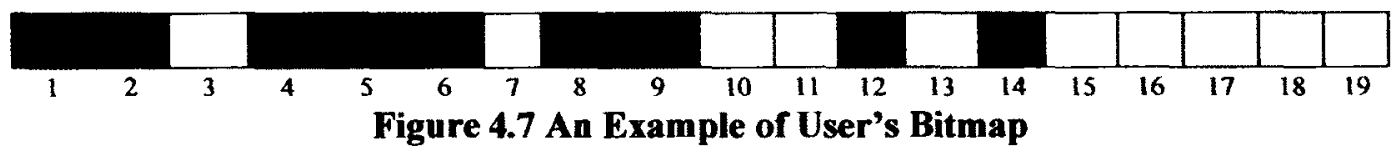

Figure 4.7 shows an example of user's bitmap. The shaded boxes indicate that the video chunks have already been downloaded, where the white boxes indicate the missing chunks. According to Definition 1, if $P(I)=90 \%$, it means that during the next downloading period, the $1^{\text {st }}$ missing chunk (chunk_3) has $90 \%$ chance to be chosen as the next downloading chunk. If $P(2)=8 \%$, it means that during the next downloading period, the $2^{\text {nd }}$ missing chunk (chunk_7) has $8 \%$ chance to be chosen as the next downloading chunk.

Note, if there are $R$ missing chunks in current bitmap (here we assume that $R$ is large enough that there is always a missing chunk that can be downloaded), then $\sum_{x=1}^{x=R} P(x)=$ 1

Definition 2. Define $W(x)$ as the probability that all downloaded chunks are sequential given $x$ chunks are already downloaded. 
For example, $W(1000)=80 \%$ means that if the user has already downloaded 1000 video chunks, then $80 \%$ of the chunks are sequential chunks. In other words, the $1^{\text {st }} 800$ chunks are all downloaded.

Definition 3. Define Sequentiality $S$ as the average percentage of sequential chunks downloaded.

For example, $S=0.9$ means that $90 \%$ of the downloaded chunks are in sequential.

According to Definition 2 and 3, we can formulate the sequentiality $S$ as:

$S=\frac{W(1)+W(2)+\ldots \ldots+W(M)}{M}$

Note: In Equation 4.1, $M$ indicates the number of downloaded chunks. We also assume the probabilities of $x=1,2,3, \ldots, M$ are uniformly distributed.

Assumption 1. $P(x)$, the probability of downloading the xth missing chunk, is geometrically distributed for MPCF algorithm, i.e. $P(x)=p(1-p)^{x-1}$, where $p$ is the probability of downloading the $I^{\text {st }}$ missing chunk (e.g. $\left.P(l)=p\right)$.

This assumption is reasonable due to the following reasons:

Firstly, by definition, the geometric distribution is the probability distribution of the Bernoulli trials needed to get one success, which is similar to our MPCF algorithm which is trying to select the first available missing chunk to download. Here, we are assuming the number of missing chunks is large enough to be treated as infinite, which is reasonable because the distribution of geometric distribution goes down very fast with increasing $x$. 
Secondly, in real-time multimedia applications, the older chunks, the chunks that are generated earlier, are always shared by more peers and therefore are more popular. Based on the definition of MPCF, peers tend to download older chunks ahead of newer chunks. As a result, the older chunks have higher probability to be downloaded. For example, if chunk index $x<y$, then $P(x)>P(y)$. This satisfies the property of geometric distribution where the probability is decreasing along the $x$-axis.

Thirdly, when the downloading process enters into steady state, the chunk selection process should be time-invariant. The memoryless property of the geometric distribution fits into this nature quite well. Based on the assumption above, we can derive the sequentiality of MPCF algorithm in the following steps:

\section{Theorem.}

$W(x)=p^{x}[1+(1-p)]\left[1+(1-p)+(1-p)^{2}\right] \ldots \ldots\left[\sum_{n=0}^{x-1}(1-p)^{n}\right]$

Proof: We will use mathematical induction to prove the above theorem.

Step 1. Let $x=1$, then substitute $x=1$ into Equation 4.2, can get:

$W(1)=p^{1}\left[1+(1-p)^{1-1}\right]=p$

$M=1$ means only 1 chunk is downloaded, then $W(1)$ is equal to the probability that this first missing chunk is downloaded, which is :

$W(1)=P(1)=p(1-p)^{0}=p$

Since Equation 4.3 and 4.4 are identical to each other, we can conclude that when $x=1$, the Theorem (Equation 4.2) is correct. 
Step 2. (Note: Step 2 is not a necessary part of the mathematical induction. However, it helps better understand the mathematical model and the proof.)

Let $x=2$, then substitute $x=2$ into Equation 4.2, can get:

$W(2)=p^{2}\left[1+(1-p)^{1-1}\right]\left[1+(1-p)^{2-1}\right]=p^{2}(1+1-p)$

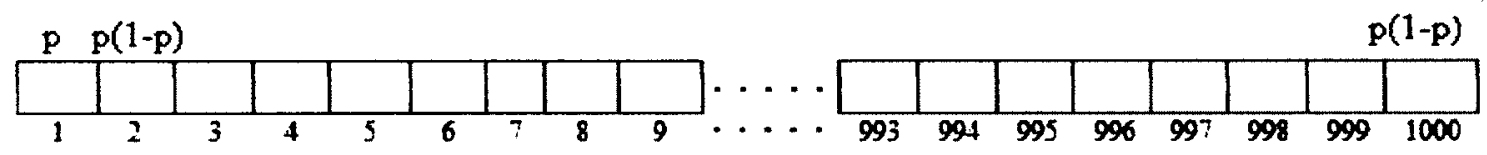

Figure 4.8 An Example of New Joined User's Bitmap

Figure 4.8 shows an example of a newly joined user's bitmap. According to our assumption, $P(1)=p, \quad P(2)=p(1-p), \ldots \ldots, P(1000)=p(1-p)^{999}$

Now, suppose 2 chunks are downloaded. We want to calculate the probability that these 2 chunks are sequential chunks, for example the probability chunk 1 and chunk 2 are downloaded. There are two possibilities:

Possibility 1: Downloading chunk 1 in first round, and downloading chunk 2 in second round. Figure 4.9 shows the situation after the first round downloading.

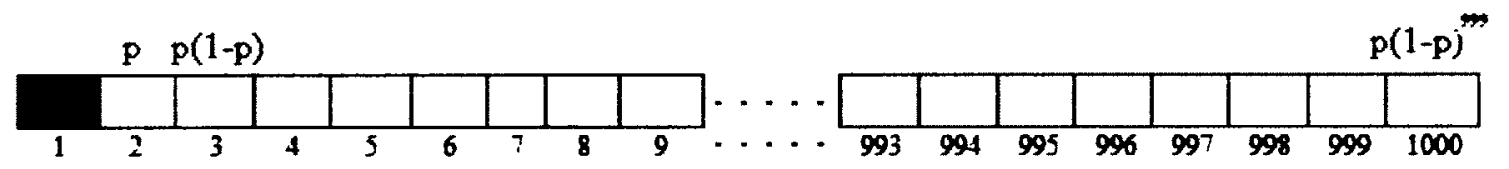

\section{Figure 4.9 User's Bitmap after Downloading the $1^{\text {st }}$ Chunk in Round 1}

The probability of downloading chunk 1 in the first round is $P(I)=p$. After the first round, the $1^{\text {st }}$ missing chunk is now chunk 2 , so the probability of downloading chunk 2 
in the second round is exactly the same as the result in Step 1, which is $P(1)$ (e.g. the probability of downloading the first missing chunk in one round). As a result, the probability of possibility 1 is equal to $P(I) \times P(I)$.

Possibility 2: Downloading chunk 2 in first round, and downloading chunk 1 in second round. Figure 4.10 shows the situation after the first round downloading.

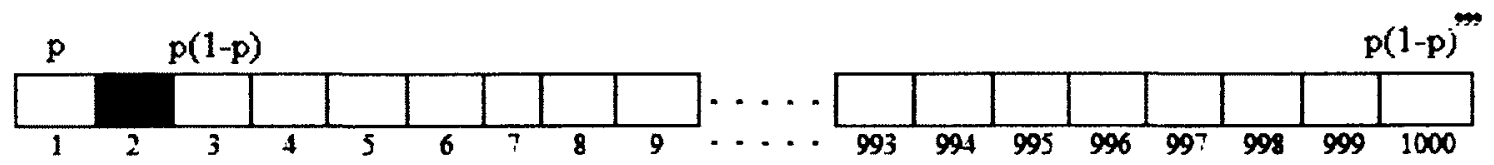

Figure 4.10 User's Bitmap after Downloading the $2^{\text {nd }}$ Chunk in Round 1

The probability of downloading chunk 2 in the first round is $P(2)=p(I-p)$. After the first round, the $1^{\text {st }}$ missing chunk is still chunk 1 , so the probability of downloading chunk 1 in the second round is still $P(1)=p$. As a result, the probability of possibility 2 is equal to $P(2) \times P(1)$.

By combining Possibility 1 and Possibility 2, the probability of downloading both chunk 1 and chunk 2 is equal to

$W(2)=P(1) P(1)+P(1) P(2)=p^{2}+p(1-p) p=p^{2}(1+1-p)$

Since Equation 4.5 and 4.6 are identical to each other, we can conclude that when $x=2$, the Theorem (Equation 4.2) is correct.

Step 3. Suppose when $x=M$, the Theorem (Equation 4.2) is correct, which can be shown as:

$W(M)=p^{M}[1+(1-p)]\left[1+(1-p)+(1-p)^{2}\right] \ldots \ldots\left[\sum_{n=0}^{M-1}(1-p)^{n}\right]$ 
Now, we will prove when $x=M+1$, the Equation 4.2 is still correct.

When $x=M+1$, it means that there are $M+1$ chunks downloaded. We want to know the possibility that all the $M+1$ chunks are sequential chunks. According to step 2 , there are $M+1$ possibilities. For example:

Possibility 1: Download the 1st missing chunk in round I, with probability $P(I)$

Possibility 2: Download the 2 nd missing chunk in round 1, with probability $P(2)$

Possibility $M+1$ : Download the $(M+1)$ th missing chunk in round 1 , with probability $P(M)$

After round 1 , the probability that the remaining $M$ chunks are sequential chunks is always $W(M)$. As a result, we have the following equations:

$$
\begin{aligned}
W(N+1) & \\
& =P(1) W(M)+P(2) W(M)+\cdots+P(M+1) W(M) \\
& =W(M) \sum_{r=1}^{M} P(r) \\
& =W(M) \sum_{r=0}^{M} p q^{r} \\
& =W(M)\left(1-q^{N+1}\right) \\
& =\left(1-q^{N+1}\right) \prod_{n=1}^{N}\left(1-q^{n}\right) \\
& =\prod_{n=1}^{M+1}\left(1-q^{n}\right)
\end{aligned}
$$

If we directly substitute $x=M+1$ into Equation 4.2 , we can get:

$$
W(M+1)=\prod_{n=1}^{M+1}\left(1-q^{n}\right)
$$


Since Equation 4.8 and 4.9 are identical to each other, we can conclude that when $x=M=1$, the Theory (Equation 4.2 ) is correct.

At this point, we have proved that the Theorem (Equation 4.2) is correct by using mathematical induction.

By observing the Equation 4.2, we find out that there is a geometric sequence inside each of the square bracket. Therefore we can use geometric series, the sum of geometric sequence $S_{n}$, to simplify the equations as the following:

$$
\begin{aligned}
W(M) & =p^{M}[1+(1-p)]\left[1+(1-p)+(1-p)^{2}\right] \ldots \ldots\left[\sum_{n=0}^{M-1}(1-p)^{n}\right] \\
& =p^{M}\left[\prod_{n=1}^{M} S_{n}\right] \quad \text { where the sum function } S_{n}=\frac{1-(1-p)^{n}}{p}
\end{aligned}
$$

The next step is to derive the Sequentiality $S$, we formulate $S$ based on all the previous results. According to definition 3, the Sequentiality can be expressed by the followings:

$$
\begin{aligned}
S & =\frac{W(1)+W(2)+\cdots+W(M)}{M} \\
& =\frac{\sum_{m=1}^{M} \prod_{n=1}^{m}\left(1-q^{m}\right)}{M}
\end{aligned}
$$

Now, we substitute $S_{n}=\frac{1-(1-p)^{n}}{p}$ into Equation 4.10, can get:

$$
\begin{aligned}
S & =\frac{1}{M} \times\left\{p+p^{2} \times \frac{[1-(1-p)]\left[1-(1-p)^{2}\right]}{p^{2}}+p^{3} \times \frac{[1-(1-p)]\left[1-(1-p)^{2}\right]\left[1-(1-p)^{3}\right]}{p^{3}}+\ldots \ldots+\right. \\
\left.p^{M} \times \frac{[1-(1-p)]\left[1-(1-p)^{2}\right] \ldots \ldots\left[1-(1-p)^{M}\right]}{p^{M}}\right\} & \\
& =\frac{1}{M} \times\left\{p+p\left[1-(1-p)^{2}\right]+p\left[1-(1-p)^{2}\right]\left[1-(1-p)^{3}\right]+\ldots \ldots+p\left[1-(1-p)^{2}\right] \ldots \ldots\left[1-(1-p)^{M}\right]\right\}
\end{aligned}
$$

Let $q=1-p$, then

$$
S=\frac{p+p\left(1-q^{2}\right)+p\left(1-q^{2}\right)\left(1-q^{3}\right)+\cdots+p\left(1-q^{2}\right)\left(1-q^{3}\right) \ldots\left(1-q^{M}\right)}{M}
$$


Equation 4.11 shows the final formulation of the sequentiality $S$, where the sequentiality $S$ can be written as a function of the number of downloaded chunks $M$, given a certain value of $p$. To test the characteristic of the sequentiality of MPCF algorithm, we use Matlab to plot the sequentiality $S$ as the function of $M$. We set $p=94.1 \%(p=94.1 \%$ is obtained from our simulation results, which will be shown in Chapter 5), for example, i.e. the probability of downloading the oldest missing chunk is $94.1 \%$, which is reasonable since the oldest missing chunk has much higher probability to be downloaded than other missing chunks. Figure 4.11 shows the plot.

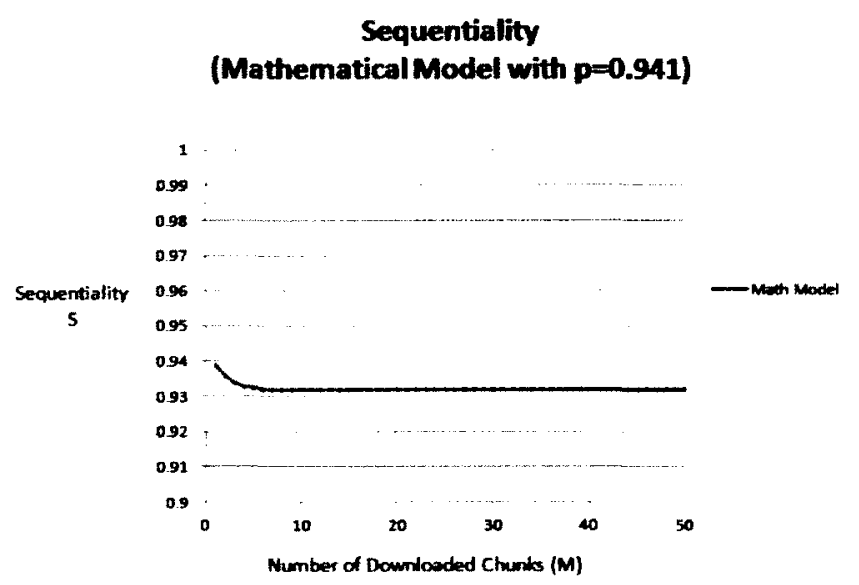

Figure 4.11 Sequentiality Vs. Number of Downloaded Chunks

As shown in Figure 4.11 , the sequentiality approaches to $S=0.932$. It means that $93.2 \%$ of the downloaded chunks are in order, which proves that MPCF algorithm can result very high sequentiality. In Chapter 5, we will show the same plot by using our simulation. Then we will compare our simulation results with the mathematical results. 


\subsubsection{Swarming Phase Implementation with MPCF}

The main idea of the swarming phase is to allow peers to exchange and download the video chunks that are missing from each other. In this section, we show the detailed design of swarming phase and how to apply MPCF algorithm to the swarming phase.

As mentioned in Chapter 3, each peer in a local group will receive random video chunks from its parent node during the diffusion phase. At the same time, swarming phase starts in the following steps:

At the beginning of the swarming phase, peers in the same local group will download the shared bitmap from the local DHT table. Here the shared bitmap is a table indicating which video chunks peers have. According to Chapter 3 , the local peers, who are close to each other, form a DHT ring structure during the registration process. Figure 4.12 shows an example of how to locate the bitmap by applying this DHT ring (Chord) structure.

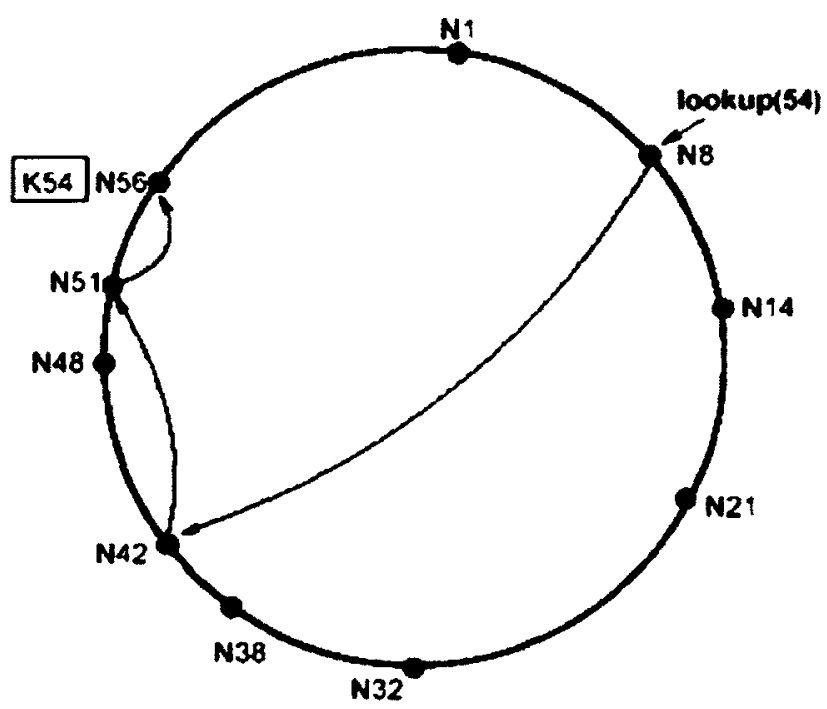

Figure 4.12 Bitmap (Key=54) Locating Process by Node 8 (N8) in Local Network 
Suppose there are 10 peers in the local network, whose IP addresses are hashed to Node ID (NID) shown in Figure 4.12 (For example, N1, N8, N14, N21, N32, N38, N42, N48, N51, and N56). Also suppose the shared bitmap is hashed to KeyID (KID) which is equal to K54. According to Chord, K54 will be stored at node N56 since N56 is the first node that satisfies $N I D \geq K I D$. In Figure 4.4, we use node $\mathrm{N} 8$ as an example. During the swarming phase, node N8 should firstly locate and download the shared bitmap each time before downloading new chunk. For N8 to locate K54, it can either pass the request clockwise along the ring (e.g. through N14, N21, N32, and so on), or use the finger table for fast key locating.

Table 4.1 and Table 4.2 show an example of the finger tables and how to locate the shared bitmap in a local network by using these finger tables.

Table 4.1 finger Table of $\mathrm{N8}$

\begin{tabular}{|c|c|}
\hline \multicolumn{2}{|c|}{ Finger Table of N8 } \\
\hline $\mathrm{N} 8+1$ & $\mathrm{~N} 14$ \\
\hline $\mathrm{N} 8+2$ & $\mathrm{~N} 14$ \\
\hline $\mathrm{N} 8+4$ & $\mathrm{~N} 14$ \\
\hline $\mathrm{N} 8+8$ & $\mathrm{~N} 21$ \\
\hline $\mathrm{N} 8+16$ & $\mathrm{~N} 32$ \\
\hline $\mathrm{N} 8+32$ & $\mathrm{~N} 42$ \\
\hline
\end{tabular}


Table 4.2 Finger Table of N42

\begin{tabular}{|c|c|}
\hline \multicolumn{2}{|c|}{ Finger Table of N42 } \\
\hline $\mathrm{N} 42+1$ & $\mathrm{~N} 48$ \\
\hline $\mathrm{N} 42+2$ & $\mathrm{~N} 48$ \\
\hline $\mathrm{N} 42+4$ & $\mathrm{~N} 48$ \\
\hline $\mathrm{N} 42+8$ & $\mathrm{~N} 51$ \\
\hline $\mathrm{N} 42+16$ & $\mathrm{~N} 1$ \\
\hline $\mathrm{N} 42+32$ & $\mathrm{~N} 14$ \\
\hline
\end{tabular}

Table 4.1 show the finger table of N8. By checking the bottom row of the table, N8 knows that node $\mathrm{N} 42$ is the closest to key K54, since 42 is the largest number which satisfies $42 \epsilon(8,54]$. Therefore N8 will directly send the request to node N42. Once N42 receives the request from $\mathrm{N} 8$, it will pass it to the closest node to key $\mathrm{K} 54$. According to Table 4.2, node N51 is the closest to K54. Therefore, the request is further passed to N51. Once N51 receives the request, it will check its finger table. Since the next node N56 satisfies $54 \epsilon(51 ; 56]$, it means that N56 is the node that stores key K54. As a result, the request is passed to N56, and the shared bitmap can be downloaded by N8. The whole locating process is shown as the curved line in Figure 4.12.

The shared bitmap is a table indicating how many copies of each video chunk are there in the local network, and which peers have the certain video chunks. Figure 4.13 shows an example of the shared bitmap. For example, the "17" in the third column means that the video chunk_3 has 17 copies in the local network. 


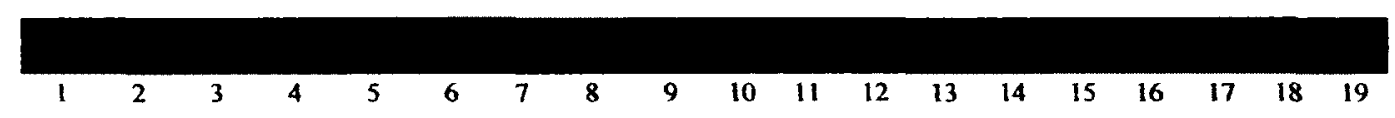

Figure 4.13 An Example of The Shared Bitmap

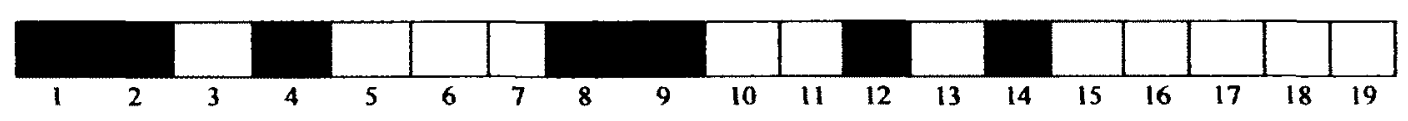

Figure 4.14 An Example of The Individual Bitmap of Node 8

By comparing the shared bitmap with its own bitmap, peer will know which video chunks are missing and which video chunk is the most popular (shared) chunk among the missing ones. For example, Figure 4.14 shows the individual bitmap of node N8, where the grey boxes indicate the chunks have already been downloaded and the white boxes indicate the missing chunks. By comparing the shared bitmap with its own bitmap, node N8 knows that chunk_3 is the missing chunk that is owned by the most number of peers in the local group. Therefore according to MPCF algorithm, N8 will choose to download chunk_3 from the one of 17 neighbours.

However if in some cases, the total uploading bandwidth for the most popular chunk is exhausted, for example in Figure 4.13, all the 17 peers who have chunk_3 do not have any available uploading bandwidth. Then the next popular chunk will be selected for downloading according to MPCF algorithm. In this case, N8 will choose to download chunk_6 first instead of waiting for chunk_3.

The shared bitmap in the local DHT table will be updated if any peer has a new chunk or deletes an old chunk. Every time a peer has the capacity to download a new chunk, it will check the local DHT table to see whether the bitmap has changed. For example, after 
node N8 downloads the chunk_6, it will update the shared bitmap as well as its own bitmap. The updated shared bitmap and individual bitmap of N8 are shown in Figure 4.15 and Figure 4.16.

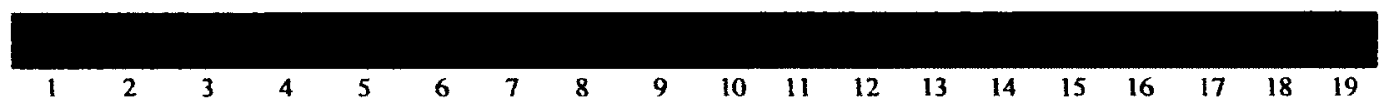

Figure 4.15 The Updated Shared Bitmap

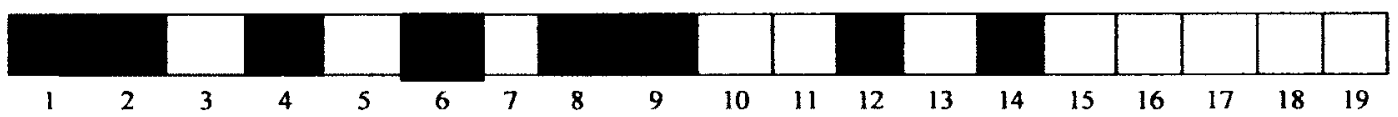

Figure 4.16 The Updated Individual Bitmap of Node 8

By applying the diffusion phase of the BU-Trie, each new peer can easily determine its position in the Trie for certain live streaming. At the same time, by applying the swarming phase, peers can easily determine which video chunks need to be downloaded and where to download the desired video chunks. If a leaf node happens to leave, only the DHT table in the lowest level group the leaf node belongs to needs to be updated. On the other hand, if a parent node at Level $\mathrm{x}$ leaves a session, another node within the same group in Level $\mathbf{x}$ will be selected immediately as the parent. Related DHT labels in the level $x+1$ to the lowest level will be updated. These DHT tables are the closest ones to the leaving node and therefore can be updated very fast. In general, the BU-Trie and the MPCF algorithm achieve our goal to localize traffic in lower level of the trie structure as much as possible and henceforth minimize delay. Each application session has its own BU-Trie. This will allow peers to share load across different live sessions and avoid bottleneck problem. 


\section{Chapter 5}

\section{Simulations and Performance Evaluations}

In this chapter, we show the OPNET simulation results and performance evaluations for the Bottom-Up Trie design. First of all, an overview of our simulation design and parameter setting are provided. In order to test the performance of P2P LVS, such as initial delay time and playback continuity, three simulations are made and provided, which include end-to-end delay test, sequential throughput test, and the inter-domain packets test. Then we compare the results with other algorithms to demonstrate the advantages of the BU-Trie design.

\subsection{Network Model}

To evaluate the performance of the BU-Trie, some simulations are designed and implemented. We use Opnet as our simulation tool. Since the initial delay time and the playback continuity are the two most important factors that reflect P2P LVS's performance, our simulations test these two factors and compare the results with other algorithms. Besides, our simulations also consider the economical efficiency for Internet service providers in terms of the inter-domain packet counts. 


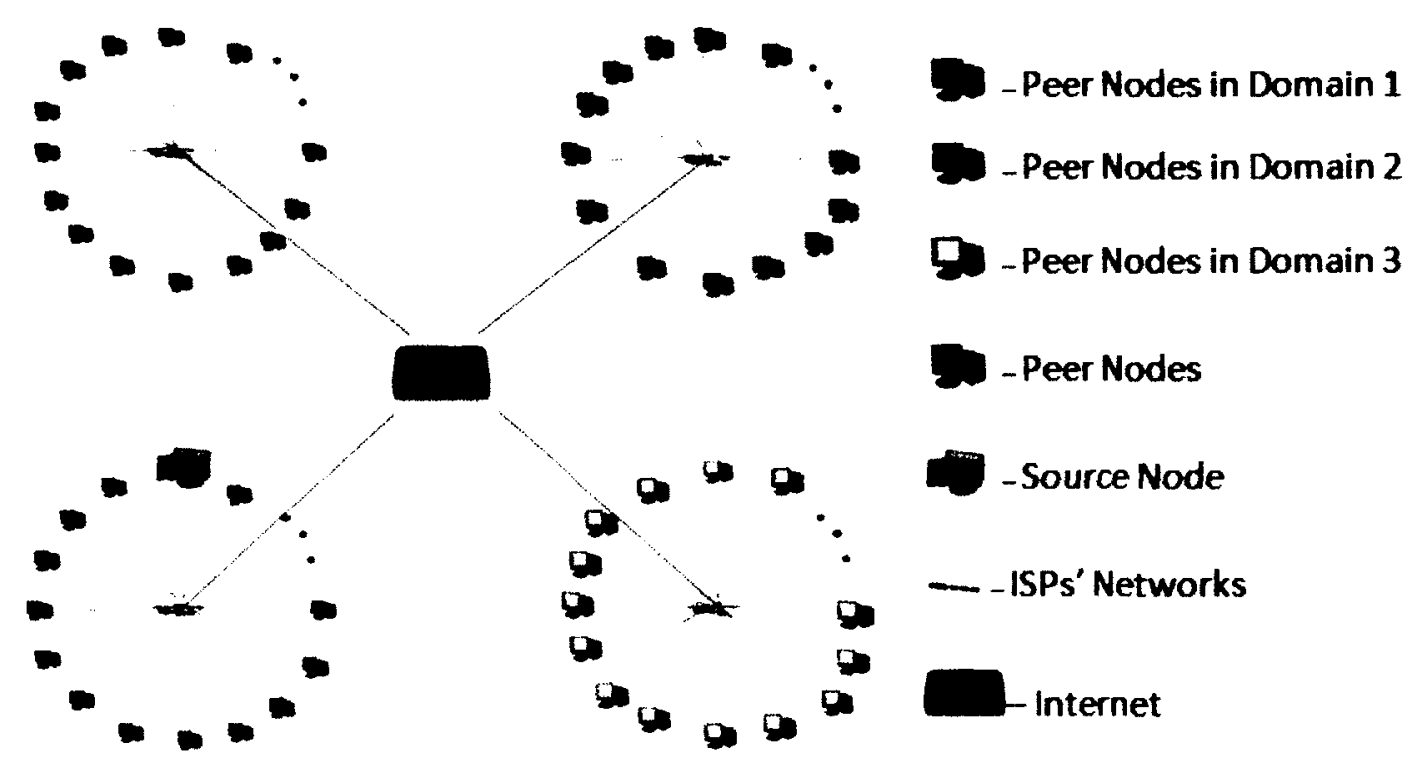

Figure 5.1 Network Topology for Simulation

For real life P2P LVS applications, there are usually thousands of users in each live streaming session. Due to the complexity and the limitation of the software, we cannot simulate such a huge number of peers. Instead, we created 128 nodes in total for our simulation. As shown in Figure 5.1, the 128 nodes are divided into 4 domains. The nodes in the same domain have same or similar IP address prefix, for example, we assume that each domain represents an ISP. We also assume that the 32 nodes in each domain are physically close to each other and have less delay. We run the simulation with heterogeneous nodes. For example, nodes have different uploading bandwidths from $5 \mathrm{Mbps}$ to $20 \mathrm{Mbps}$. Our simulation focuses on one live streaming session. Therefore one node is selected as the source node as shown in Figure 5.1. The source node keeps generating live video chunks, where each chunk has unique size of $512 \mathrm{~KB}$. In this 
simulation, we build a 3-level $B U-T r i e$, where we set $H=28, M=16$. Figure 5.2 shows the BU-Trie structure.

Each level 1 ring has 8 peers, where the 8 peers have the same higher 28 bits in their IP addresses. One of the 8 peers in each ring is then selected as the parent peer node. These 16 parent nodes form the level 2 rings based on the higher 16 bits in their IP addresses. After that, 4 level 2 parent nodes are selected from the 4 level 2 rings, and form the level 3 ring. Therefore the level 3 ring has 4 parent nodes, and one of them is the source node.

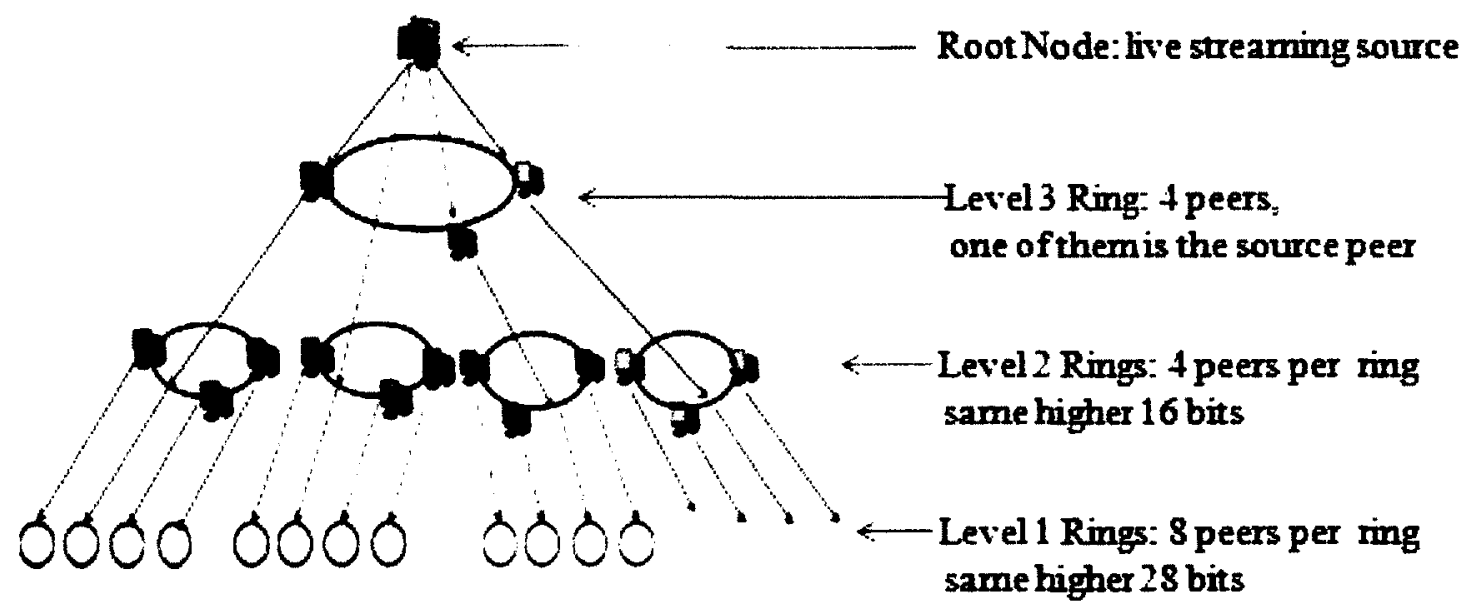

Figure 5.2 BU-Trie Structure for Simulation

Three tests are made with this simulation. Test 1 is the Sequential throughput test. Sequential throughput is defined as the number of sequential chunks downloaded per second. In other words, we measure the average downloading speed of the in-order video chunks. For P2P LVS applications, in order to have smooth video playback, the Sequential throughput must be larger than or at least equal to the video playback rate. Otherwise, there will be pauses during the video playback. So higher sequential throughput means better playback continuity. Test 2 is the end-to-end (ETE) delay test. The ETE delay is measured by the waiting time from the time of sending one chunk by 
the source node to the time of receiving this chunk by a leaf node. Shorter ETE delay means less initial buffering time. Test 3 measures the average number of inter-domain packets, i.e., the average number of packets that are transmitted between different domains. We compare the results with three other algorithms: Rarest-First (RF), Naive Sequential (NS), and Hybrid of RF and NS (half RF half NS).

\subsection{Sequential Throughput Test}

Simulation 1 is the sequential throughput test. In this simulation, we record the total number of chunks received by the node and the total number of chunks in sequence (sequential chunks) during the simulation. Then we use the data collected to analyze the performance of the design. Without loss of generality, we randomly pick a node (node_6) to show our analysis as an example. Then we repeat the same analysis for other nodes in the same domain and further analyze the results.

\subsubsection{Bottom-Up Trie (BU-Trie)}

First of all, BU-Trie Design is tested. We export the graphic data obtained from Opnet to Excel, and obtain the following plots as shown in Figure 5.4, 
Number of Packets Received Vs. Time

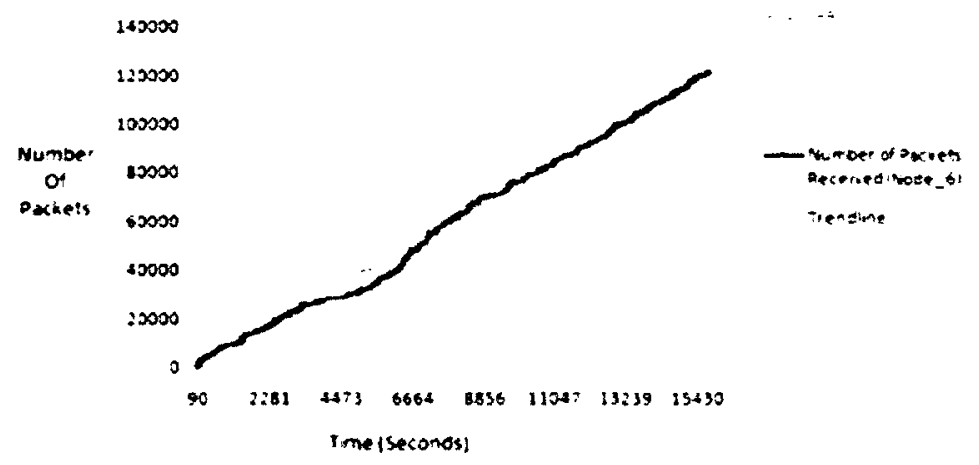

(A)

Number of Sequential Packets Vs. Time

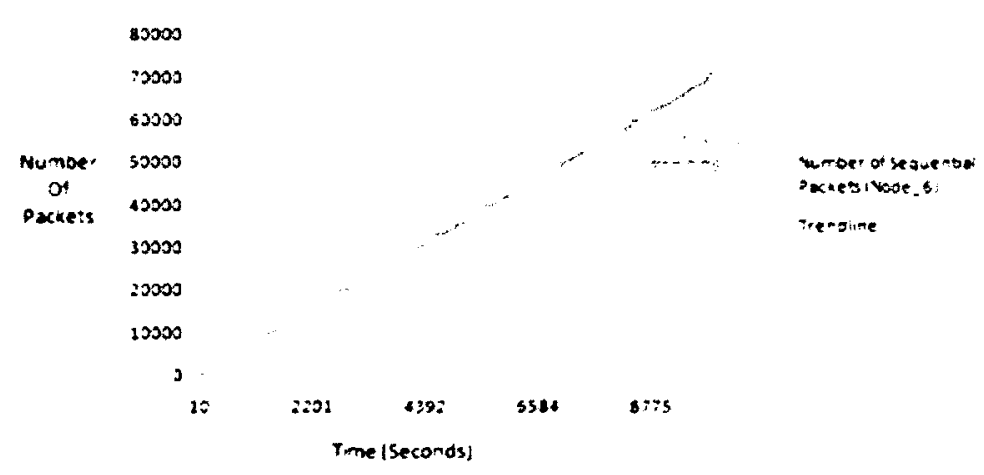

(B)

Figure 5.3 Number of Packets Received Vs. Time (BU-Trie)

\section{(A) total number of packets received (B) total number of sequential packets received}

As shown in Figure 5.3, the red line represents the total number of packets received by node 6 versus time (Fig $5.3(\mathrm{~A})$ ). The orange line represents the total number of packets received sequentially versus time (Fig 5.3 (B)). The blue lines represent the trendlines of 
the two plots. As discussed previously, the slops of the trendlines represent the average throughput (TR) and the average sequential throughput (STR). In this test, we get

$$
\left\{\begin{array}{l}
\text { TR }=7.54 \text { packets } / \mathrm{sec} \\
\text { STR }=7.06 \text { packets } / \mathrm{sec}
\end{array}\right.
$$

As stated, the packets size is $512 \mathrm{~KB}$. Therefore, equation can be represented as

$$
\left\{\begin{array}{l}
\text { TR }=3.86 \mathrm{Mbps} \\
\mathrm{STR}=3.61 \mathrm{Mbps}
\end{array}\right.
$$

The above analysis is based on the simulation results obtained from the random picked node (node 6). Next, we repeat this analysis for the rest 31 nodes in domain 1 . The throughput and sequential throughput of all the 32 peers are shown in Figure 5.4.

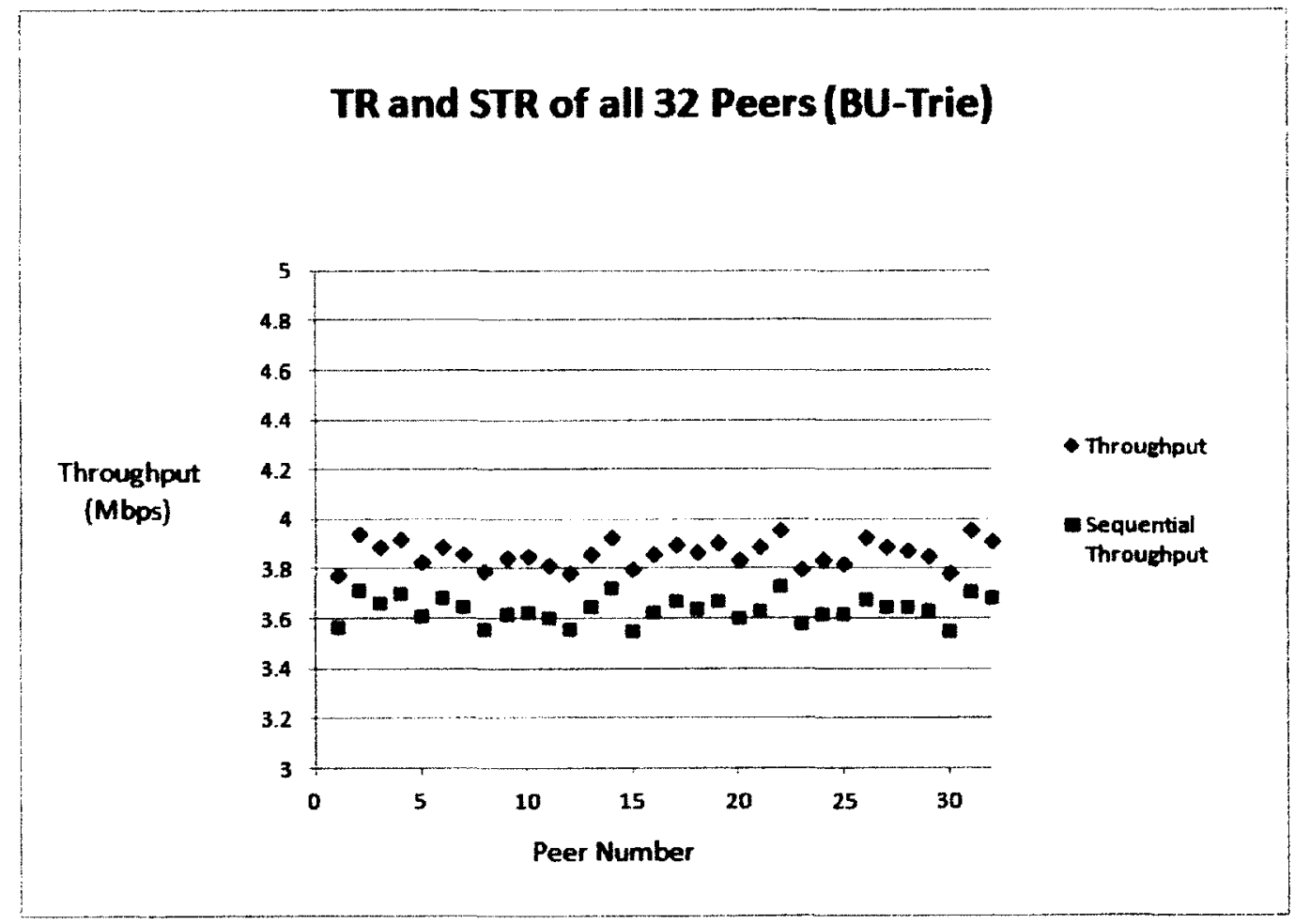

Figure 5.4 Throughputs and Sequential Throughputs of 32 Peers (BU-Trie) 
Then we calculate the average value and confidential interval for both throughput and sequential throughput from the data shown in Figure 5.4, Table 5.1 shows the results we got.

Table 5.1 Simulation Results and Confidential Interval (BU-Trie)

\begin{tabular}{|c|c|c|}
\hline & Average Value (Mbps) & $95 \%$ Confidential Interval \\
\hline Throughput (TR) & 3.85 & $(3.83,3.87)$ \\
\hline Sequential Throughput (STR) & 3.61 & $(3.59,3.63)$ \\
\hline
\end{tabular}

By using the average value shown in Table 5.1, we can calculate the sequentiality as the following,

$$
\text { Sequentiality }=\frac{3.61}{3.85}=0.938
$$

The sequentiality obtained from the simulation means that $93.8 \%$ of the packets are downloaded sequentially, which is consistent with our design described in Chapter 4.

Next we repeat this sequential throughput test for 3 other algorithms, which are RarestFirst (RF), Naive Sequential (NS), and Hybrid (HB, e.g. half RF half NS).

\subsubsection{Rarest-First (RF)}

According to Chapter 4, RF algorithm is where peers always download the rarest-shared chunks from the available peers. Figure 5.5 shows the sequential throughput test results of the RF algorithm. Without loss of generality, we also choose the randomly picked node (node_6) to show our analysis as an example. 
Number of Packets Received Vs. Time

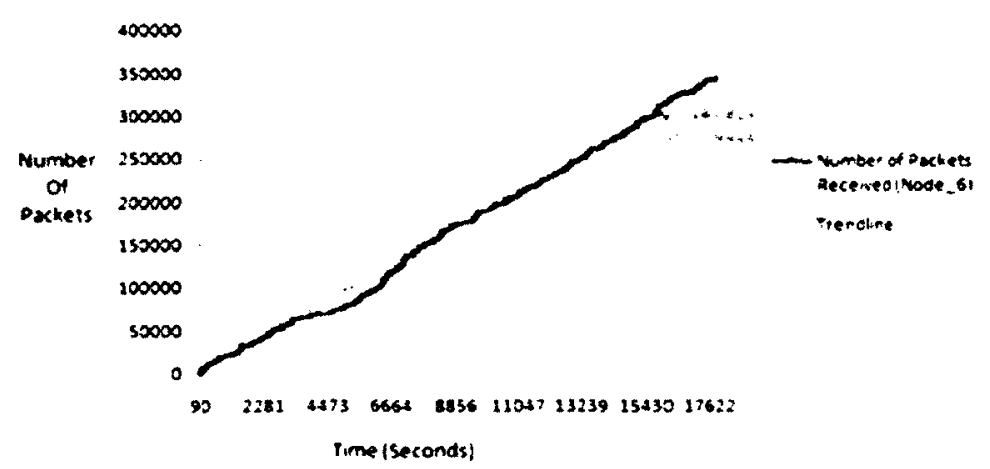

(A)

Number of Sequential Packets Vs. Time

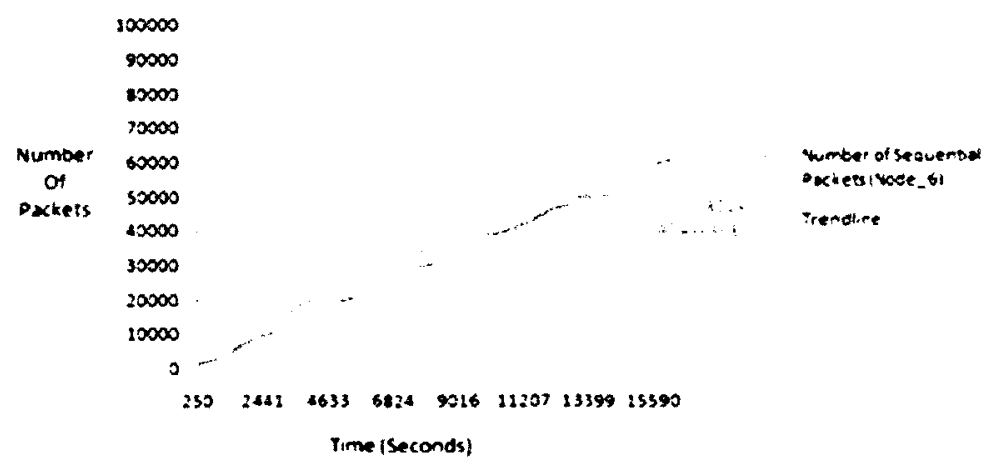

(B)

Figure 5.5 Number of Packets Received Vs. Time (BU-Trie)

(A) Total number of packets received (B) Total number of sequential packets received

As shown in Figure 5.5, the red line represents the total number of packets received by node 6 versus time (Fig 5.5 (A)). The orange line represents the total number of packets received sequentially versus time (Fig $5.5(\mathrm{~B})$ ). The blue lines represent the trendlines of 
the two plots. As discussed previously, the slops of the trendlines represent the average throughput (TR) and the average sequential throughput (STR). In this test, we get

$$
\left\{\begin{array}{l}
\mathrm{TR}=19.08 \text { packets } / \mathrm{sec} \\
\mathrm{STR}=3.78 \text { packets } / \mathrm{sec}
\end{array}\right.
$$

As stated, the packets size is $512 \mathrm{~KB}$. Therefore, equation can be represented as

$$
\left\{\begin{array}{l}
\text { TR }=10.12 \mathrm{Mbps} \\
\text { STR }=1.93 \mathrm{Mbps}
\end{array}\right.
$$

The above analysis is based on the simulation results obtained from the random picked node (node_6). Next, we repeat this analysis for the rest 31 nodes in domain 1 , The throughput and sequential throughput of all the 32 peers are shown in Figure 5.6

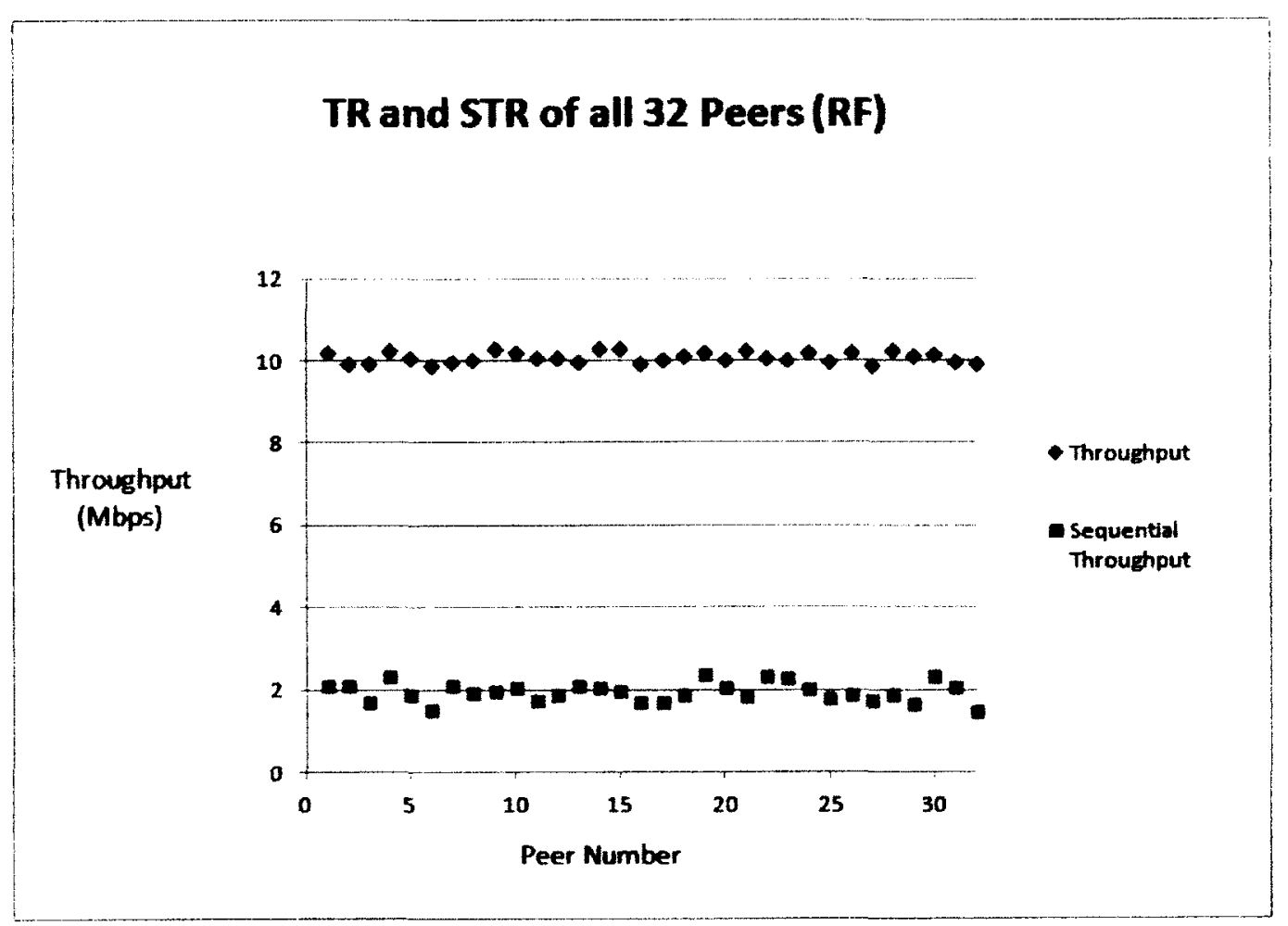

Figure 5.6 Throughputs and Sequential Throughputs of 32 Peers (RF) 
Then we calculate the average value and confidential interval for both throughput and sequential throughput from the data shown in Figure 5.6, Table 5.2 shows the results we got.

Table 5.2 Simulation Results and Confidential Interval (RF)

\begin{tabular}{|c|c|c|}
\hline & Average Value (Mbps) & $95 \%$ Confidential Interval \\
\hline Throughput (TR) & 10.08 & $(9.59,10.58)$ \\
\hline Sequential Throughput (STR) & 1.91 & $(1.82,2.01)$ \\
\hline
\end{tabular}

By using the average value shown in Table 5.2, we can calculate the sequentiality as the following,

$$
\text { sequentiality }=\frac{1.91}{10.08}=0.19
$$

The sequentiality obtained from the simulation means that $19 \%$ of the chunks are downloaded sequentially. Most downloaded chunks are not in sequence, which is consistent with our design described in Chapter 4.

\subsubsection{Naive Sequential (NS)}

According to Chapter 4, NS algorithm is where peers always download the next chunk that is in order according to the chunk number. Figure 5.7 shows the sequential throughput test results of the NS algorithm. Without loss of generality, we also choose the randomly picked node (node 6) to show our analysis as an example. 
Number of Packets Received Vs. Time

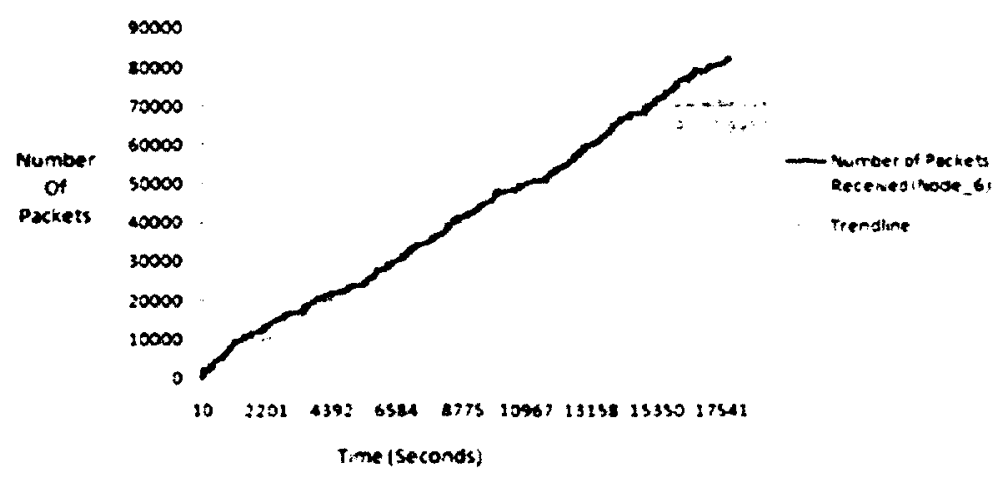

(A)

Number of Sequential Packets Vs. Time

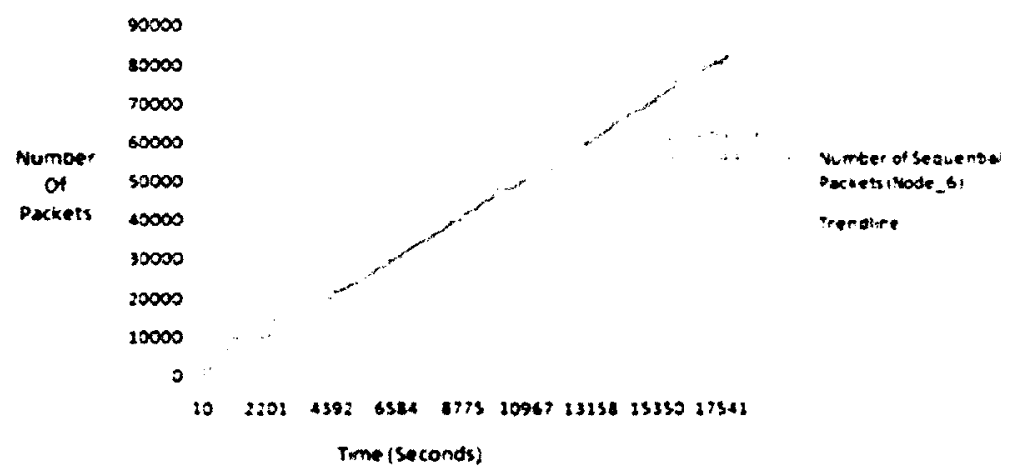

(B)

Figure 5.7 Number of Packets Received Vs. Time (NS)

(A) Total number of packets received (B) Total number of sequential packets received

As shown in Figure 5.7, the red line represents the total number of packets received by node_6 versus time (Figure $5.7(\mathrm{~A})$ ). The orange line represents the total number of packets received sequentially versus time (Figure 5.7 (B)). The blue lines represent the 
trendlines of the two plots. As discussed previously, the slops of the trendlines represent the average throughput (TR) and the average sequential throughput (STR). In this test, we get

$$
\left\{\begin{aligned}
\mathrm{TR} & =4.64 \text { packets } / \mathrm{sec} \\
\mathrm{STR} & =4.64 \text { packets } / \mathrm{sec}
\end{aligned}\right.
$$

As stated, the packets size is $512 \mathrm{~KB}$. Therefore, equation can be represented as

$$
\left\{\begin{array}{c}
\text { TR }=2.37 \mathrm{Mbps} \\
\text { STR }=2.37 \mathrm{Mbps}
\end{array}\right.
$$

The above analysis is based on the simulation results obtained from the random picked node (node_6). Next, we repeat this analysis for the rest 31 nodes in domain 1 , the throughput and sequential throughput of all the 32 peers are shown in Figure 5.8

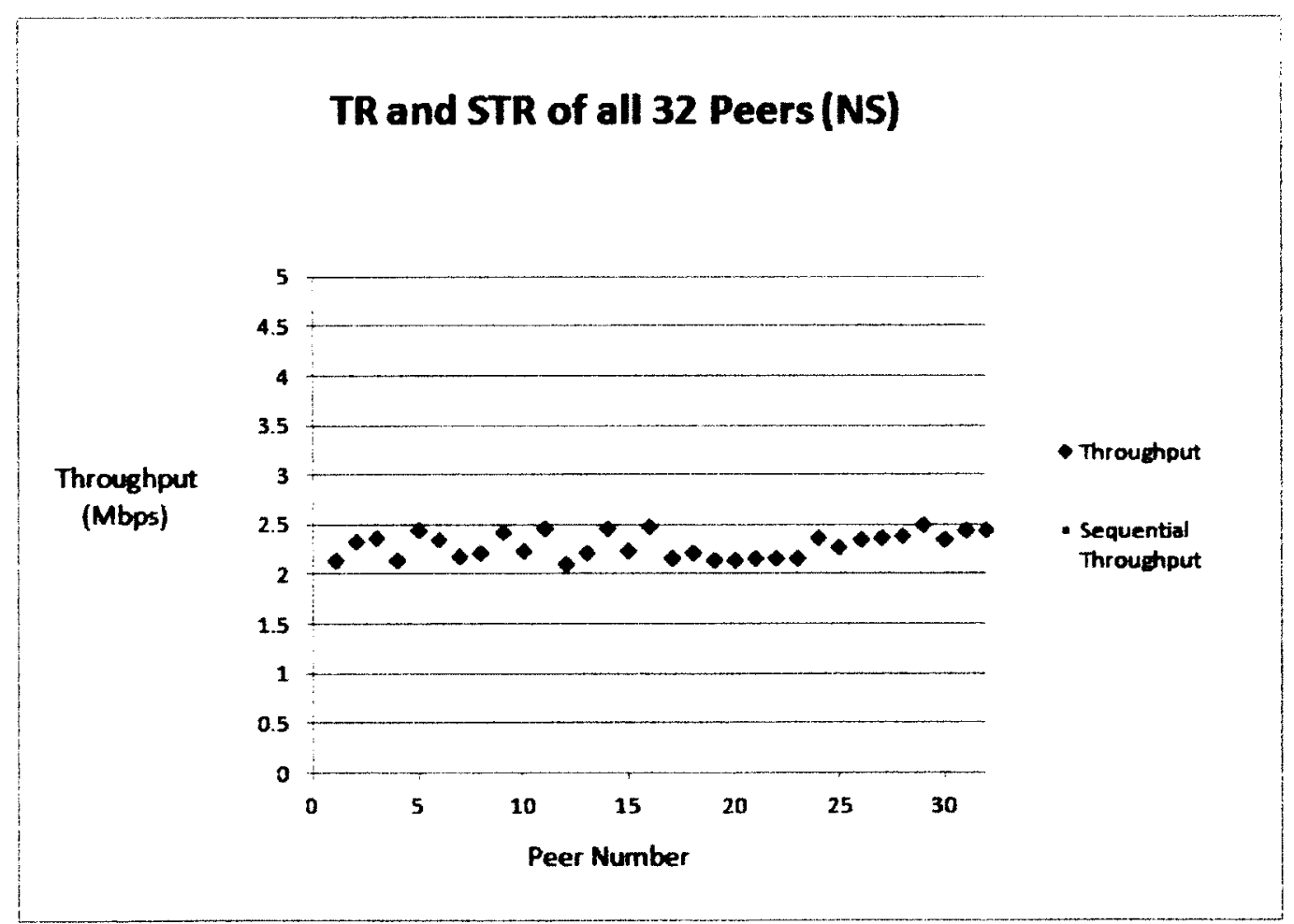


Figure 5.8 Throughputs and Sequential Throughputs of 32 Peers (NS)

Then we calculate the average value and confidential interval for both throughput and sequential throughput from the data shown in Figure 5.8, Table 5.3 shows the results we got.

Table 5.3 Simulation Results and Confidential Interval $(s=1)$

\begin{tabular}{|c|c|c|}
\hline & Average Value (Mbps) & 95\% Confidential Interval \\
\hline Throughput (TR) & 2.30 & $(2.18,2.41)$ \\
\hline Sequential Throughput (STR) & 2.30 & $(2.18,2.41)$ \\
\hline
\end{tabular}

By using the average value shown in Table 5.8, we can calculate the sequentiality as the following,

$$
\text { sequentiality }=\frac{2.30}{2.30}=1
$$

The equation 5.12 shows that sequentiality obtain from the simulation means that $100 \%$ of the packets are downloaded sequentially, which is exactly our setting value. We can conclude that our simulation results are accurate and acceptable.

\subsubsection{Hybrid (HB)}

According to definition, HB algorithm is where peers download half of the chunks by RF algorithm and half of the chunks by NS. Figure 5.9 shows the sequential throughput test results of the $\mathrm{HB}$ algorithm. Without loss of generality, we also choose the randomly picked node (node 6) to show our analysis as an example. 
Number of Packets Received Vs. Time

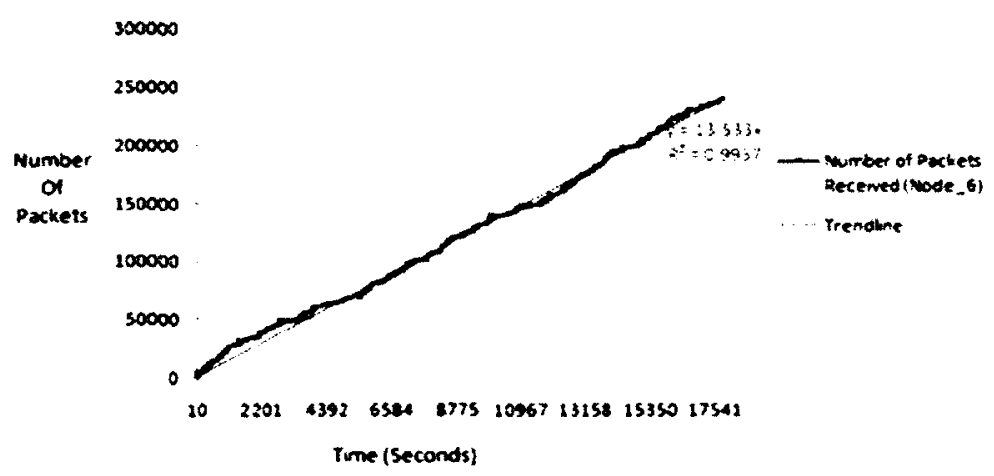

(A)

\section{Number of Sequential Packets Vs. Time}

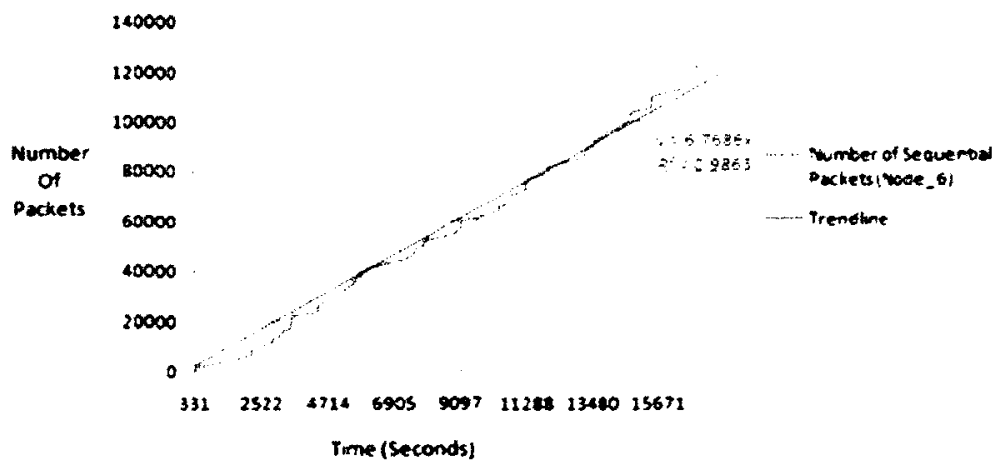

(B)

Figure 5.9 Number of Packets Received Vs. Time (HB)

(A) Total number of packets received (B) Total number of sequential packets received

As shown in Figure 5.9, the red line represents the total number of packets received by node 6 versus time (Figure 5.9 (A)). The orange line represents the total number of packets received sequentially versus time (Figure 5.9 (B)). The blue lines represent the 
trendlines of the two plots. As discussed previously, the slops of the trendlines represent the average throughput (TR) and the average sequential throughput (STR). In this test, we get

$$
\left\{\begin{array}{l}
\mathrm{TR}=13.53 \text { packets } / \mathrm{sec} \\
\mathrm{STR}=6.76 \text { packets } / \mathrm{sec}
\end{array}\right.
$$

As stated, the packets size is $512 \mathrm{~KB}$. Therefore, equation can be represented as

$$
\left\{\begin{array}{c}
\mathrm{TR}=6.92 \mathrm{Mbps} \\
\mathrm{STR}=3.46 \mathrm{Mbps}
\end{array}\right.
$$

The above analysis is based on the simulation results obtained from the random picked node (node_6). Next, we repeat this analysis for the rest 31 nodes in domain 1 , the throughput and sequential throughput of all the 32 peers are shown in Figure 5.10

\section{TR and STR of all 32 Peers (HB)}

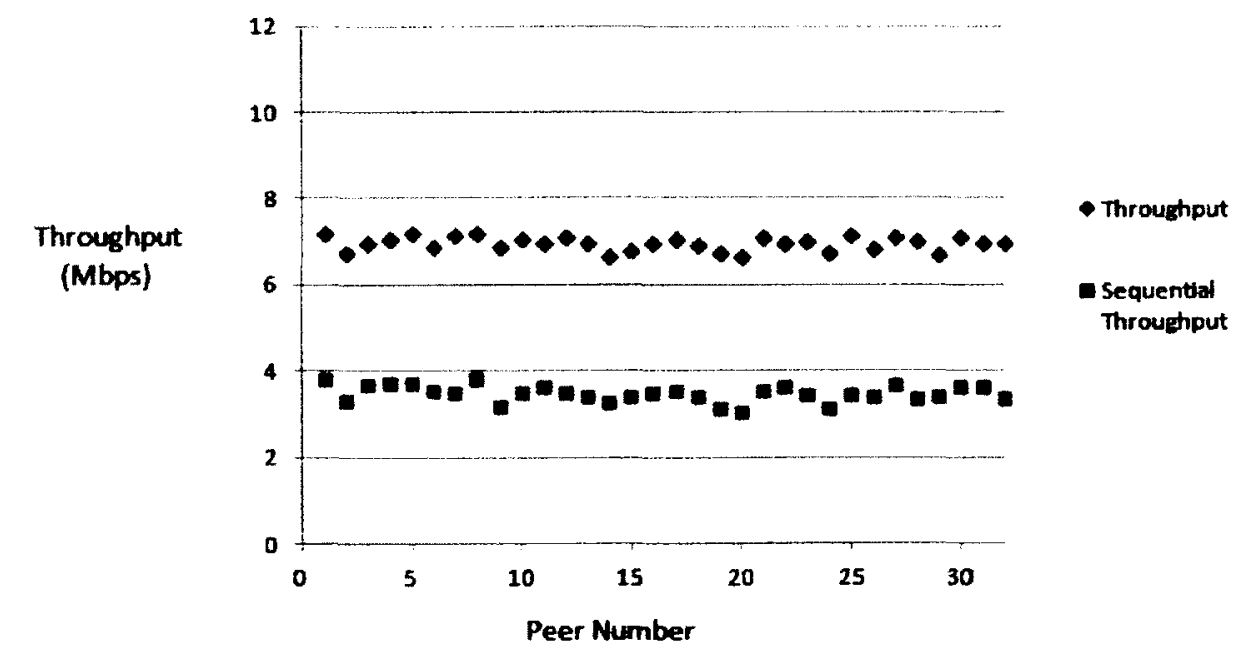

Figure 5.10 Throughputs and Sequential Throughputs of 32 Peers (HB) 
Then we calculate the average value and confidential interval for both throughput and sequential throughput from the data shown in Figure 5.10, Table 5.5 shows the results we got.

Table 5.4 Simulation Results and Confidential Interval (HB)

\begin{tabular}{|c|c|c|}
\hline & Average Value (Mbps) & $95 \%$ Confidential Interval \\
\hline Throughput (TR) & 6.91 & $(6.77,7.06)$ \\
\hline Sequential Throughput (STR) & 3.41 & $(3.22,3.60)$ \\
\hline
\end{tabular}

By using the average value shown in Table 5.10, we can calculate the sequentiality as the following,

$$
\text { sequentiality }=\frac{3.41}{6.91}=0.494
$$

The equation shows that sequentiality obtained from the simulation means that $49.4 \%$ of the packets are downloaded sequentially, which is close to our setting value (50\%).

\subsubsection{Results Comparison}

In this section, we compare the results obtained from previous 4 simulations. First of all, we combine the 4 simulation results together (which are shown in Figures 5.3, 5.5, 5.7, and 5.9), in order to show the graphical comparison of the 4 algorithms. 


\section{Average Total Throughput Test}

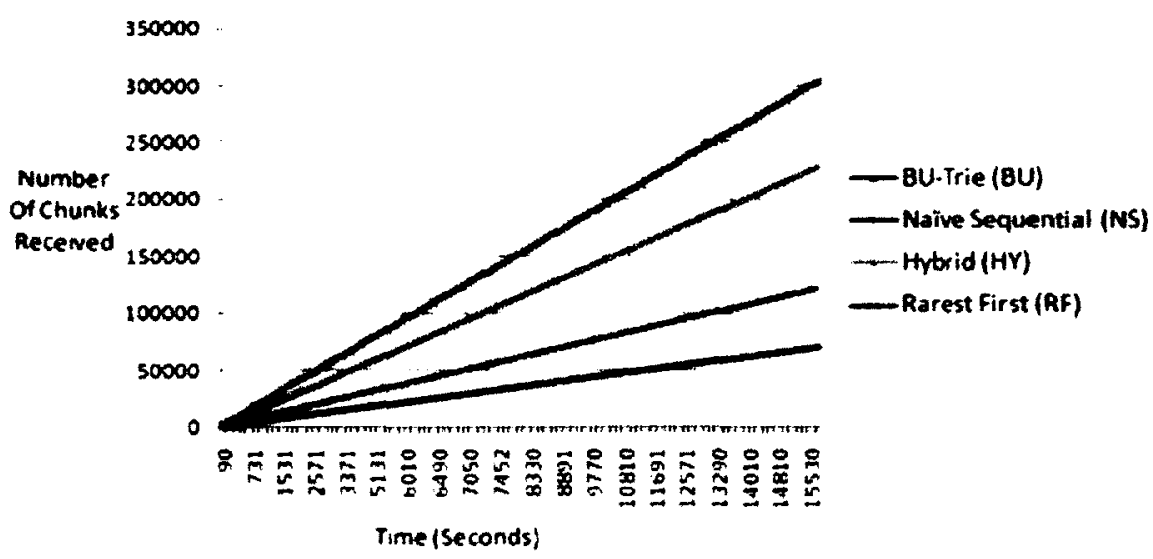

(A)

\section{Average Sequential Throughput Test}

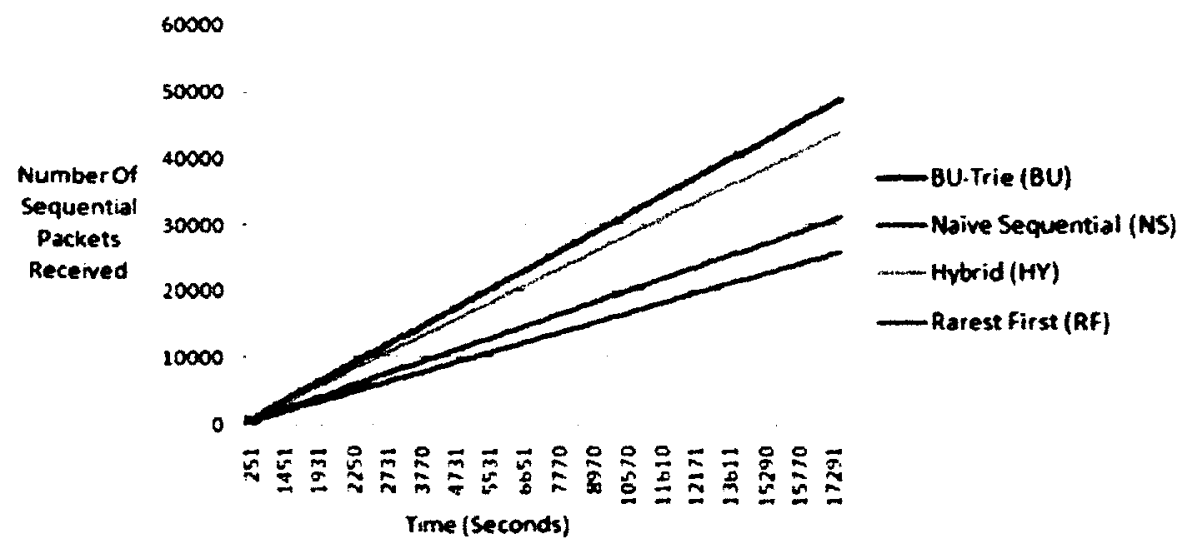

(B)

Figure 5.11. Number of Chunks Received Vs. Time (Comparison)

(A) Total number of chunks received (B) Number of sequential chunks received 
As shown in Figure 5.11, the red line, blue line, green line, and purple line represent the number of packets received by node_6 versus time when applying BU-Trie, RF, NS, and HB respectively.

For the total throughput test (Figure $5.11(\mathrm{~A})$ ), from the slope of each line, we can see that the throughput ranking from the highest to the lowest is: RF, HB, BU-Trie, and NS. This result is exactly what we expected, since the RF algorithm lets the system optimize the even distribution of all chunks which optimize the average downloading speed, and NS algorithm makes the chunk distribution heavily bias toward early chunks which results a low downloading speed as discussed in Chapter 4 . However, for the sequential throughput test (Figure 5.11 (B)), the BU-Trie design achieves the highest sequential throughput as predicted since it can both relatively high throughput and sequentiality.

To get a more clear comparison, we combine the numerical results (which are shown in Tables 5.1, 5.2, 5.3, 5.4) and show it in Table 5.5.

Table 5.5 Sequential Throughput Test Results (Comparison)

\begin{tabular}{|l|l|l|l|}
\hline Test & $\begin{array}{l}\text { Average } \\
\text { Throughput } \\
\text { (Mbps) }\end{array}$ & $\begin{array}{l}\text { Sequential } \\
\text { Throughput } \\
\text { (Mbps) }\end{array}$ & Sequentiality \\
\hline BU & 3.85 & 3.61 & 0.938 \\
\hline RF & 10.09 & 1.91 & 0.19 \\
\hline NS & 2.30 & 2.30 & 1 \\
\hline HY & 6.91 & 3.41 & 0.494 \\
\hline
\end{tabular}


Table 5.5 shows the average throughput and average sequential throughput by using the slope of the Figure 5.11 multiplied with $512 \mathrm{k}$ (the size of each chunk). We can see that even though the Rarest-First algorithm has the highest total throughput, the chunks downloaded are not in order. As a result, the playback continuity is poor due to the low sequential throughput. For Naive Sequential algorithm, all downloaded chunks are in order, but the throughput is relatively low since most of the peers download the newest chunk at the same time. BU-Trie has higher total throughput than the Naive Sequential and much higher sequentiality than the Rarest-First. As a result, BU-Trie achieves the highest sequential throughput as predicted.

\subsubsection{Comparison of Simulation Results and Mathematical Model}

Another test is to compare the sequentiality of BU-Trie that obtained by the mathematical model (Figure 4.11) with our simulation results. The simulation results can be obtained from the data shown in Figure 5.4, where we can use the average sequential throughput divided by the average total throughput. In order to use our mathematical model, we collect the probability of downloading the $1^{\text {st }}$ missing chunk in each downloading round. We repeat the simulation 10 times, and got the following results.

Table 5.6 The Average Probability of Downloading the $1^{\text {st }}$ Missing Chunks in Each Round

\begin{tabular}{|c|c|c|c|c|c|c|c|c|c|c|}
\hline Simulation & 1 & 2 & 3 & 4 & 5 & 6 & 7 & 8 & 9 & 10 \\
\hline$p$ & 0.945 & 0.943 & 0.939 & 0.941 & 0.937 & 0.943 & 0.939 & 0.939 & 0.942 & 0.946 \\
\hline
\end{tabular}

From table 5.6, we can calculate the average $p=0.941$, which will be used in our mathematical model as the value of $p$. 
Figure 5.12 shows the comparison results of the sequentiality of BU-Trie that obtained by the mathematical model $(p=0.94 I)$ with our simulation results:

\section{Sequentiality Comparison \\ (Mathematical Model with p $=0.941$ V. Simulation)}

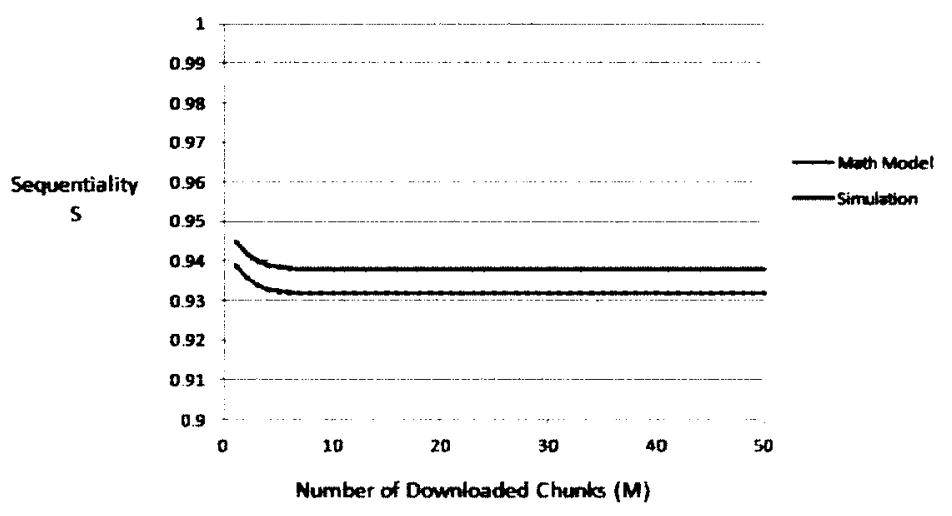

Figure 5.12 Sequentiality Comparison Test: Math Model Vs. Simulation

As shown in Figure 5.12, the average sequentiality obtained from mathematical model is equal to $S=0.932$, where the average sequentiality obtained from simulation is equal to $S=0.938$. Both of the two plots approach to certain value as $M$, the number of downloaded chunks, increases. Both the values of $S$ and the shapes of the plots are close to each other, for example, the value of sequentiality decreases as $M$ increases and approaches to a fixed value when $M$ reaches certain value. which prove that our simulation result is acceptable.

In order to test the characteristic of MPCF algorithm, we repeat the above simulation test by decreasing the uploading speed. For example, the uploading bandwidth of each peer node is decreased by $30 \%$. All other settings are kept same as before. The following figures shows the results we get from this test. 


\section{TR and STR of all 32 Peers (BU-Trie)}

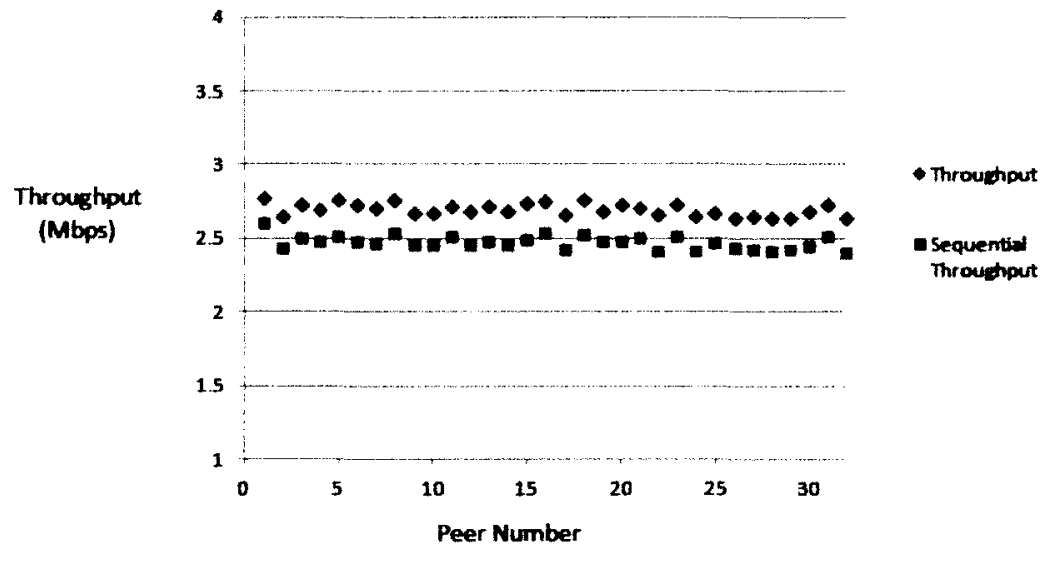

Figure 5.13 Throughputs and Sequential Throughputs of 32 Peers

(Lower Uploading Bandwidth)

Figure 5.13 shows average throughput and sequential throughput of 32 peers after decreasing the uploading bandwidth. Then we calculate the average value and confidential interval for both average throughput and sequential throughput from the data shown in Figure 5.13, Table 5.7 shows the results we got.

\begin{tabular}{|c|c|c|}
\hline & Average Value (Mbps) & $95 \%$ Confidential Interval \\
\hline Throughput (TR) & 2.72 & $(2.68,2.76)$ \\
\hline Sequential Throughput (STR) & 2.45 & $(2.41,2.49)$ \\
\hline
\end{tabular}

Table 5.7 Simulation Results and Confidential Interval (BU-Trie)

By comparing Table 5.7 with Table 5.1, we can see that the average throughput is decreased by $(3.85-2.73) / 3.85=29.1 \%$, and the average sequential throughput is decreased by $(3.61-2.45) / 3.61=32.1 \%$. The results are reasonable since the overall 
downloading speed of the network is decreased $30 \%$. Now we collect the probability of downloading the $1^{\text {st }}$ missing chunk in each downloading round. And we get the average probability of downloading the $1^{\text {st }}$ missing chunk in each downloading round is $p=0.910$. Then substitute $p=0.910$ into our mathematical model, and plot the sequentiality as the function of the number of downloaded chunks. Then we compare the sequentiality that obtained by the mathematical model with our simulation results.

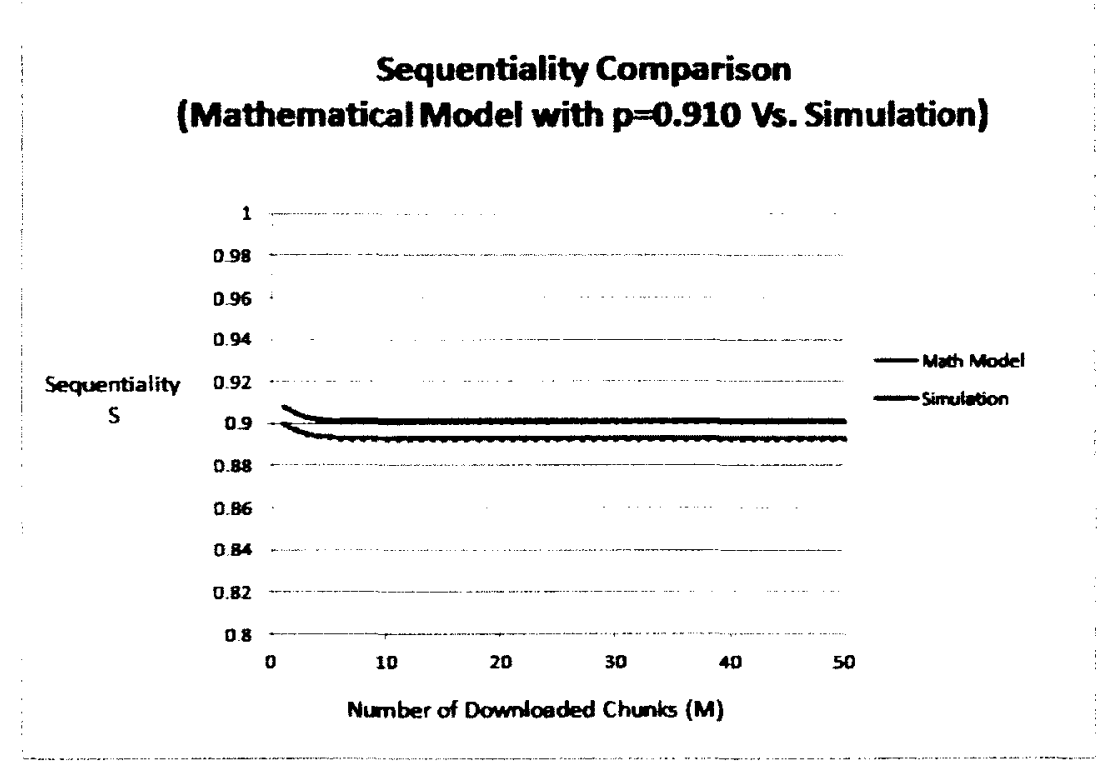

Figure 5.14 Sequentiality Comparison Test: Math Model Vs. Simulation

As shown in Figure 5.14, the average sequentiality obtained from mathematical model is equal to $S=0.893$, where the average sequentiality obtained from simulation is equal to $S=0.901$. Both of the two plots approach to certain value as $M$, the number of downloaded chunks, increases. Both the values of $S$ and the shapes of the plots are close to each other, which prove that our simulation result is acceptable. The reason that the sequentiality of MPCF algorithm decreases is because the most popular chunks may not have enough uploading bandwidth for all peers to download, so the next popular chunks 
are selected according to our algorithm. However, the sequentiality is still very high even though the uploading bandwidth is lower.

\subsection{End-to-End Delay Test}

Test 2 is the end-to-end (ETE) delay test. For each chunk generated by the source node, we record the time when chunk is generated and the time when chunk is successfully received by the client. Then the ETE delay is measured by the difference of these 2 recorded times. Obviously shorter ETE delay can result less initial buffering time as discussed in previous chapter. Figure 5.15 shows the ETE delay of each chunk received by the randomly picked node 6 .

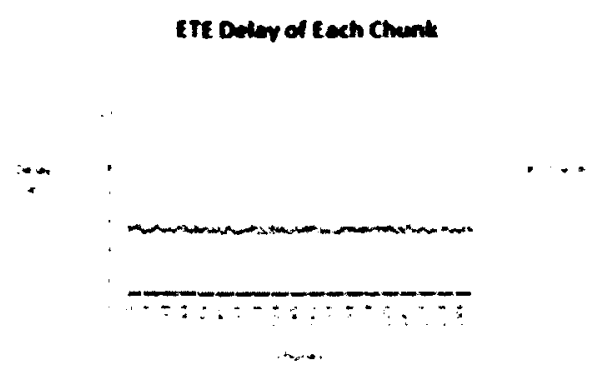

(A)

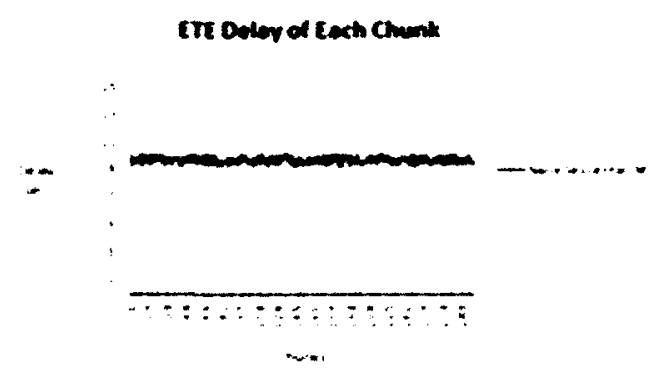

(C)

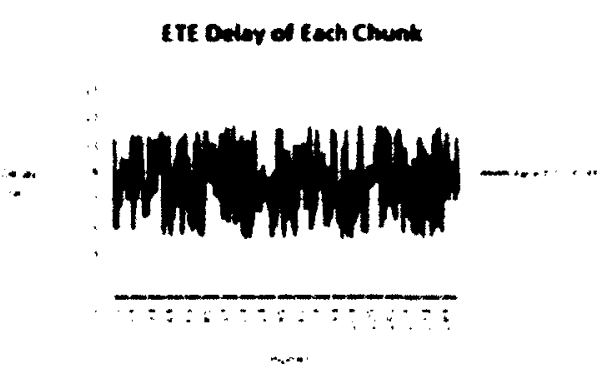

(B)

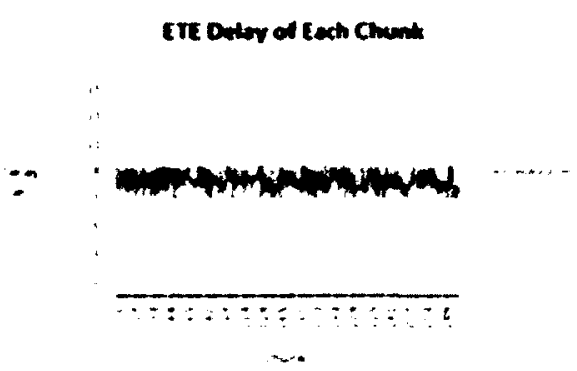

(D)

Figure 5.15 End-to-End Delay of Each Video Chunk
(A) ETE Delay of BU-Trie
(B) ETE Delay of Rarest-First
(C) ETE Delay of Naive Sequential (D) ETE Delay of Hybrid 
For RF algorithm (Figure 5.15 (B)), the rarest-shared chunks have the smallest ETE delay, and those chunks are usually the newest generated chunks. NS algorithm has relatively stable ETE delay (Figure 5.15 (C)), but the delay is high due to the limited uploading bandwidth of the newest chunk. For Hybrid algorithm (Figure 5.15 (D)), the chunks downloaded by RF algorithm have low and unstable ETE delay. The chunks downloaded by NS algorithm have high and stable ETE delay as expected. For BU-Trie ((Figure 5.15 (A)), the ETE delay is relatively low and stable. The delay is stable, because it has similar downloading order as the NS algorithm. However, the delay of BU-Trie is much lower, this is due to: 1. MPCF algorithm makes sure the next downloaded chunk always has the largest uploading bandwidth, and it allows peers to move on to the next popular chunk if there is a bandwidth bottleneck. 2. The BU-Trie allow peers to exchange data with the peers that are close to each other, for example, within the same ISP or physically close to each other. As a result, the propagation delay is much lower.

\subsection{Inter-Domain Chunks Test}

Test 3 is made to count the average number of the inter-domain packets during 1 hour live streaming session. We repeat the test 10 times for each algorithm, and the results are shown in Figure 5.16 and Table 5.8. The reason we make this test is because P2P applications always generate huge amount inter-domain traffic between different ISPs, and as a result ISPs usually do not prefer users to use P2P-based software. 


\section{Number of Inter-Domain Chunks}

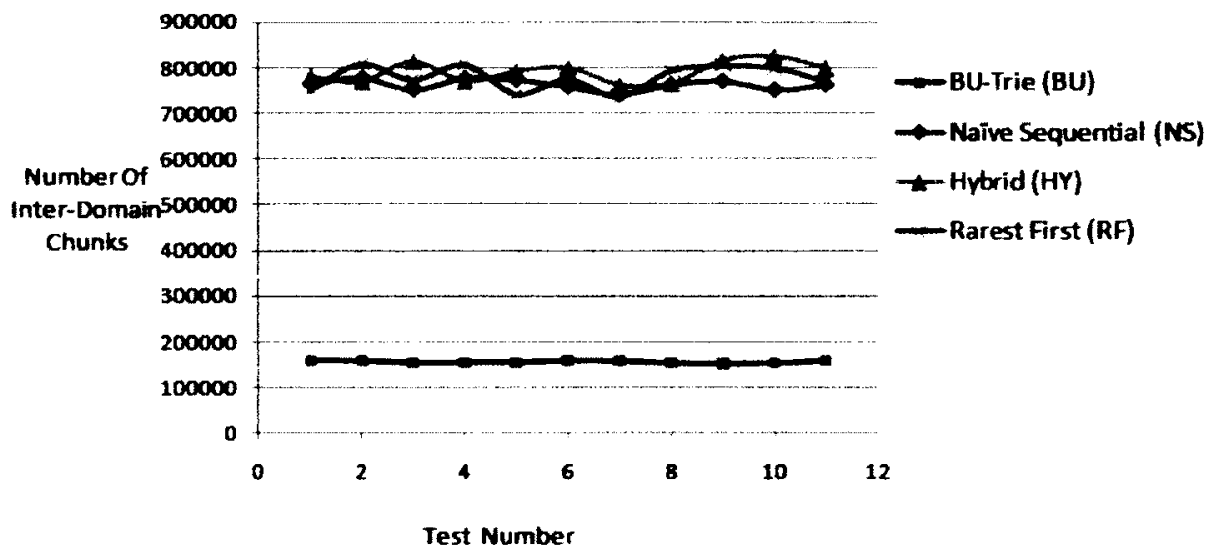

Figure 5.16. Number of Inter-Domain Video Chunks

Table 5.8 Average Number of Inter-Domain Video Chunks

\begin{tabular}{|c|c|}
\hline Algorithm & $\begin{array}{c}\text { Average Number of Inter-Domain } \\
\text { Chunks }\end{array}$ \\
\hline BU & 15612 \\
\hline RF & 76270 \\
\hline NS & 80334 \\
\hline HY & 76412 \\
\hline
\end{tabular}

From the graphical results in Figure 5.16 and numerical results shown in Table 5.8, the number of inter-domain packets is reduced significantly by applying the BU-Trie design. Since most of the traffic in the lower level of the BU-Trie is within the same domain, the traffic between different ISPs is much less as predicted. 


\section{Chapter 6}

\section{Conclusion}

\subsection{Conclusion}

Although the current existing P2P LVS software supports a large variety of live channel selection, end users have experienced problems such as non-smooth playing back during the time watching live content. The reasons behind these problems are lack of uploading bandwidth, unbalanced throughput and sequentiality relationship. Previous researches introduced different architectures of building P2P network for video streaming applications. However, these researches didn't consider the physical locations of peers when building the P2P network. Besides, most of researches apply the combination of Rarest-First and Naive Sequential as the video chunk selection algorithm, which still result low sequential throughput due to the weaknesses of these two algorithms. To our best knowledge, there is no such study that can improve the overall performance for $\mathrm{P} 2 \mathrm{P}$ LVS application as well as localize the traffic as much as possible. In this case, the goal of our research is to design a $\mathrm{P} 2 \mathrm{P}$ architecture for organizing peers, and to design an algorithm for optimizing data exchanging among those peers, in order to improve both throughput and sequentiality and finally achieve smooth play-back for P2P LVS. 
Therefore, we design a multibit Trie structure called Bottom-Up Trie (BU-Trie) for P2P live streaming. Different from other tree-based structure, BU-Trie has two phases: diffusion phase and swarming phase. The diffusion phase is where peers form the BUTrie structure and source node push the streaming data to certain peers. The swarming phase is where the peers download streaming data from each other.

The purpose of diffusion phase is to group the local peers together and design the path from the video source to all peers. By doing this, the source node can distribute streaming data as widely as possible to each local domain. The main design goal of diffusion phase is to minimize the initial end-to-end delay for distributing live contents from source to end users by discovering the physical locations of the peers and connecting peers who are close to each others in terms of physical locations. For each live streaming session, a BUTrie is formed by all the peers who are willing to download the live content before the actual data transmission. Meanwhile, the BU-Trie is dynamic and will adjust its structure according to existing peers' absence and new peers' presence.

The swarming phase of the BU-Trie starts right after the diffusion phase. For the swarming phase, the main objective is to find an optimal way for distributing the video stream within a local network. We show that by applying the proposed Most Popular Chunk First (MPCF) algorithm to the swarming phase, the sequential throughput of downloaded chunks is increased compared to other approaches. The gain in throughput would result in better playback continuity and shorter start-up delay.

In order to show the performance of our BU-Trie design, we design several simulations to test and verify our approach. From the simulation result, we conclude that the 
sequential throughput can be increased, since the BU-Trie can achieve both relatively high throughput and sequentiality. The end-to-end delay can be reduced as a result of fast distribution of data and peer localization. We compared our design with three other existing algorithms. The comparison results demonstrate the advantages of BU-Trie over other algorithms.

\subsection{Suggestions for Future Work}

In order to be applied in real P2P network, other simplified parameters or the parameters that are not shown in our design, such as downloading bandwidth limit, processing delay, and so on, need to be considered. The existing parameters, such as uploading bandwidth, also need to be treated as dynamic variable instead of fixed value. The optimization function will then depend on more variables. In the future, the mathematical model can be re-formulated based on the new involved parameters.

The simulation work can also be improved by comparing with more existing models of P2P live streaming network. For the testing of our own design, a larger scale can be introduced to involve more peers to the simulated network. Moreover, the parallel downloading can also be considered, where there exists several different video source that distributing parallel video streaming to different groups of users. 


\section{References}

[1] L. Guo, S. Chen, Z. Xiao, E. Tan, X. Ding, X. Zhang, "A performance study of BitTorrent-like peer-to-peer systems," Selected Areas in Communications, IEEE Journal on, vol.25, no.1, pp.155-169, 2007.

[2] PPLive, http://www.pptv.com/en/.

[3] UUSee, http://www.uusee.com/.

[4] SopCast, http://www.sopcast.com/.

[5] X. Liu, H. Yin, C. Lin, Y. Liu, Z. Chen, X. Xiao, "Performance analysis and industrial practice of peer-assisted content distribution network for large-scale live video streaming." 22nd International Conference on Advanced Information Networking and Applications (aina 2008), pp.568-574, Okinawa, March 2008.

[6] B. Fan, D. Andersen, M. Kaminsky, K. Papagiannaki, "Balanceing Throughput, Robustness, and In-Order Delivery in P2P VoD, " ACM Conference on emerging Networking EXperiments and Technologie (CoNEXT 2010), Philadelphia, November 2010.

[7] S. Banerjee, B. Bhattacharjee, and C. Kommareddy, "Scalable Application Layer Multicast," ACM Special Interest Group on Data Communications (SIGCOMM 2002), pp. 205-217, vol. 32, no. 4, Pittsburgh, August 2002. 
[8] Tran, D.A.; Hua, K.A.; Do, T.; , "ZIGZAG: an efficient peer-to-peer scheme for media streaming," IEEE International Conference on Computer Communications (INFOCOM 2003), pp. 1283- 1292, vol. 2, San Francisco, March 2003.

[9] N. Padmanabhan, J. Wang, A. Chou, and K. Sripanidkulchai, "Distributing Streaming Media Content Using Cooperative Networking,". Network and Operating System Support for Digital Audio and Video (NOSSDAV 2002), Miami, May 2002.

[10] Z. Lu, Y. Li, J. Wu, S. Zhang, Y. Zhong, "MultiPeerCast: A tree-mesh-hybrid P2P live streaming scheme design and implementation based on PeerCast." 10th IEEE International Conference on High Performance Computing and Communications (HPCC 2008), pp.714-719, Dalian, September 2008.

[11] C. Xu, G. M. Muntean, E. Fallon, A. Hanley, "A balanced tree-based strategy for unstrucatured media distribution in P2P networks." IEEE International Conference on Communications (ICC 2008), Beijing, May 2008.

[12] H. Liu, I.Wu, F.Jen, "MeTree: A Contribution and Locality-Aware P2P Livee Streaming Architecture." Advanced Information Netowrking and Application (ANIA 2010), pp.1136-1143, Perth, March 2010.

[13] X.Tu, H.Jin, X.Liao, J.Cao, "Nearcast: A locality-aware P2P live streaming approach for distance education." ACM Transctions on Internet Technology (ACM TOIT 2008), vol. 8 Issue 2, New York, February 2008.

[14] V. N. Padmanabhan, H. J. Wang, and P. A. Chou, "Resilient peer-to-peer streaming," International Classification for Nursing Practice (ICNP 2003), Georgia, November 2003. 
[15] M. Castro, P. Druschel, A.-M. Kermarrec, A. R. A. Nandi, and A. Singh, "SplitStream: High-bandwidth content distribution in a cooperative environment," ACM Symposium on Operating Systems Principles (ACM SOSP 2003), New York, Octorber 2003.

[16] Y. Cui and K. Nahrstedt. Layered peer-to-peer streaming. 13th ACM Network and Operating System Support for Digital Audio and Video (ACM NOSSDAV 2003). pp. 162-171, Monterey, June 2003.

[17] L. Guo, E. Tan, S. Chen, Z. Xiao, O. Spatscheck, and X. Zhang, "Delving into internet streaming media delivery: A quality and resource utilization perspective," ACM Special Interest Group on Data Communication (ACM SIGCOMM 2006), Pisa, September 2006.

[18] T. Small, B. Liang, and B. Li, "Scaling laws and tradeoffs of peer-to-peer live multimedia streaming," ACM Multimedia 2006, Santa Barbara, October 2006.

[19] S. Tewari and L. Kleinrock, "Analytical model for bittorrent-based live video streaming. IEEE Networking Issues in Multimedia Entertainment (IEEE NIME 2007) Workshop, New York, January 2007.

[20] E. Veloso, V. Almeida, W. Meira, A. Bestavros, and S. Jin. "A hierarchical characterization of a live streaming media workload," IEEE/ACM Transactions on Networking, Vol.14, No.1, February 2006.

[21] A. Vlavianos, M. Iliofotou, and M. Faloutsos. "Bitos: Enhancing bittorrent for supporting streaming applications," IEEE International Conference on Computer Communications (IEEE INFOCOM 2006), Barcelona, April 2006. 
[22] C. Wu and B. Li. "Optimal peer selection for minimum-delay peer-to-peer streaming with rateless codes," ACM Workshop on Advances in P2P Multimedia Streaming, Hilton, November 2005.

[23] G. Wu and T. cker Chiueh, "How efficient is bittorrent?" Society of Photographic Instrumentation Engineers (SPIE 2006), Orlando, May 2006.

[24] M. Zhang, L. zhao, Y. Tang, J.-G. Luo, and S.-Q. Yangt, "Large-scale live media streaming over peer-to-peer networks through global internet," International Multimedia Conference Proceedings of the ACM workshop on Advances in peer-topeer multimedia streaming, Hilton, November 2005.

[25] X. Zhang, J. Liu, B. Li, and T.-S. P. Yum, "Coolstreaming/donet: A data-driven overlay network for efficient live media streaming," IEEE International Conference on Computer Communications (IEEE INFOCOM 2005), Miami, March 2005.

[26] N. Magharei, R. Rejaie, and Y. Guo, "Mesh or multiple-tree: A comparative study of live P2P streaming approaches." IEEE International Conference on Computer Communications (IEEE INFOCOM 2007), Anchorage, May 2007.

[27] N. Magharei, R. Rejaie, "PRIME: Peer-toPeer Receiver-Driven Mesh-Based Streaming”, IEEE/ACM transactions on networking, vol. 17, no. 4, August 2009.

[28] K. Sripanidkulchai, "The Feasibility of Supporting Large-scale Live Streaming Applications with Dynamic Application EndPoints", ACM Special Interest Group on Data Communication (ACM SIGCOMM 2004), Portland, August 2004.

[29] X. Zhang; J. Liu; B. Li; Y.-S.P.; , "CoolStreaming/DONet: a data-driven overlay network for peer-to-peer live media streaming," IEEE International Conference on Computer Communications (IEEE INFOCOM 2005), Miami, March 2005. 
[30] Stoica, I.; Morris, R.; Liben-Nowell, D.; Karger, D.R.; Kaashoek, M.F.; Dabek, F.; Balakrishnan, H. , "Chord: a scalable peer-to-peer lookup protocol for Internet applications," IEEE/ACM Transactions on Networking, vol.11, no.1, pp. 17- 32, Feb 2003.

[31] Opnet, http://www.opnet.com/

[32] S. Asaduzzaman, Y. Qiao, G. Bochmann, "CliqueStream: An Efficient and Faultresilient Live Streaming Network on a Clustered Peer-to-peer Overlay," Eight Interatioanl Conference on Peer-to-Peer Computing (P2P'08), Aachen, 2008.

[33] Y. Zhou, D. Chiu, J. Lui, "A Simple Model for Analyzing P2P Streaming Protocols," IEEE International Conference on Network Protocols (IEEE ICNP 2007), Beijing, 2007.

[34] B. Zhao, J. Lui, D. Chiu, "Exploring the Optimal Chunk Selection Policy for Data-Driven P2P Streaming Systems," The 9th Conference on Peer-to-Peer Computing, 2009.

[35] L. Dai, Y. Cui, and Y. Xue, "Maximizing throughput in layered peer-to-peer streaming," IEEE International Conference on Communications (IEEE ICC 2007), Glasgow, 2007.

[36] M.-T. Lu, J.-C. Wu, K.-J. Peng, P. Huang, J. J. Yao, and H. H.Chen, “Design and evaluation of a P2P IPTV system for heterogeneousnetworks," IEEE Trans. Multimedia, vol. 9, no. 8, pp. 1568-1579, 2007.

[37] H. Guo, K.-T. Lo, Y. Qian, and J. Li, "Peer-to-peer live video distributionunder heterogeneous bandwidth constraints," IEEE Trans. Parallel Distrib. Syst., vol. 20, no. 2, pp. 233-245, Feb. 2009. 
[38] M.-T. Lu, H. Nien, J.-C. Wu, K.-J. Peng, P. Huang, J. J. Yao, C.-C. Lai, and H. H. Chen, "A scalable peer-to-peer IPTV system," 4TH IEEE Consumer Communications and Networking Conference (CCNC 2007), pp. 313-317, Las Vegas, 2007.

[39] A. Sentinelli, G. Marfia, M. Gerla, L. Kleinrock, and S. Tewari, "WillIPTV ride the peer-to-peer stream? [peer-to-peer multimedia streaming]," IEEE Commun. Mag., vol. 45, no. 6, pp. 86-92, June 2007.

[40] X. Hei, C. Liang, J. Liang, Y. Liu, and K. W. Ross, "A measurementstudy of a large-scale P2P IPTV system," IEEE Trans. Multimedia, vol. 9, no. 8, pp. 1672$1687,2007$.

[41] C. Yang, Z. Zhong, "A P2P streaming media application layer multicast technology under the constraint of delay and bandwidth," Consumer Electronics, Communications and Networks (CECNet 2011), pp.3847-3850, Xianning, 2011

[42] H.Byun, M. Lee, "A tracker-based P2P system for live multimedia streaming services," International Conference on Advanced Communication Technology (ICACT 2011), pp.1608-1613, 13-16 Feb. Phoenix Park, 2011.

[43] B. Zhang, S.G.Chan, G.Cheung, E.Y.Chang, "LocalTree: An Efficient Algorithm for Mobile Peer-to-Peer Live Streaming," IEEE International Conference on Communicaitions (ICC 2011), pp.1-5, 5-9, Kyoto, 2011.

[44] Y. Zhao, H. Shen, "A simple analysis on P2P streaming with peer playback lags," IEEE 3rd International Conference on Communication Software and Networks (ICCSN 2011), pp.396-400, 27-29, Xi'an, 2011. 
[45] C. Lan; H. Lu; C.Yi, C Tseng, "A P2P HD Live Video Streaming system," International Conference on Multimedia Technology (ICMT 2011), volpp.475-478, 26-28, Hangzhou, 2011.

[46] H. Wehbe, G. Babonneau, B. Cousin, "Mesh-based overlay enhancing live video quality in pull-based P2P systems," IEEE 3rd International Conference on Communication Software and Networks (ICCSN 2011), pp.326-332, 27-29, Xi'an, 2011.

[47] M. J. Freedman, M. Vutukuru, N. Feamster, H. Balakrishnan, "Geographic locality of IP Prefixes." ACM International Measurement Conference (ACM IMC 2005), Berkeley,October 2005. 